\title{
A Meta-Analysis of Placebo-Controlled Clinical Trials Assessing the Efficacy and Safety of Incretin-Based Medications in Patients with Type 2 Diabetes
}

\author{
Walid K.H. Fakhoury Corinne LeReun Donna Wright \\ IMS Health, London, UK
}

\section{Key Words}

Incretin mimetics - Dipeptyl peptidase type 4 inhibitor .

Diabetes $\cdot$ Glycemic control $\cdot$ Hypoglycemia $\cdot$ Weight

\begin{abstract}
Aims: A systematic review of the literature, in combination with a meta-analysis of randomized controlled trials comparing treatments with placebo, was conducted to provide an update on the clinical efficacy and safety of incretinbased medications in adult patients with type 2 diabetes. Methods: A literature search (2000-2009) identified 38 placebo-controlled trials (phase II or later - parallel design) comparing exenatide $(n=8)$, liraglutide $(n=7)$, vildagliptin $(n=11)$ and sitagliptin $(n=12)$ with placebo. Outcomes were change from baseline in $\mathrm{HbA}_{1 \mathrm{c}}$ and in weight, and the number of patient-reported hypoglycemic episodes. $\mathrm{HbA}_{1 \mathrm{c}}$ and weight outcomes were analyzed as weighted mean differences (WMD), and the number of hypoglycemic episodes as relative risks (RR). Results: Patients receiving liraglutide showed greater reduction in $\mathrm{HbA}_{1 \mathrm{c}}$ in comparison to placebo (WMD $=-1.03,95 \%$ confidence interval, $\mathrm{Cl}=-1.16$ to -0.90 , $\mathrm{p}<0.001)$ than those on sitagliptin (WMD $=-0.79,95 \% \mathrm{Cl}=$ -0.93 to $-0.65, \mathrm{p}<0.001$ ), exenatide (WMD $=-0.75,95 \% \mathrm{Cl}=$ -0.83 to $-0.67, \mathrm{p}<0.001$ ) or vildagliptin (WMD $=-0.67,95 \%$ $\mathrm{Cl}=-0.83$ to $-0.52, \mathrm{p}<0.001)$. Weight was statistically sig-
\end{abstract}

nificantly negatively associated with exenatide (WMD = $-1.10,95 \% \mathrm{Cl}=-1.32$ to $-0.87, \mathrm{p}<0.001)$ and positively associated with sitagliptin $(\mathrm{WMD}=0.60,95 \% \mathrm{Cl}=0.33-0.87, \mathrm{p}<$ 0.001 ) and vildagliptin (WMD $=0.56,95 \% \mathrm{Cl}=0.27-0.84, \mathrm{p}<$ $0.001)$. The number of patient-reported hypoglycemic episodes was statistically significantly associated with the use of sitagliptin ( $R R=2.56,95 \% \mathrm{Cl}=1.23-5.33, \mathrm{p}=0.01)$ and exenatide ( $R R=2.40,95 \% \mathrm{Cl}=1.30-4.11, \mathrm{p}=0.002)$. Conclusion: Incretin-based therapies are effective in glycemic control and also offer other advantages such as weight loss (exenatide and liraglutide). This may have an important impact on patient adherence to medication.

Copyright $\odot 2010$ S. Karger AG, Basel

\section{Introduction}

Diabetes is a serious global health issue, with type 2 diabetes mellitus (T2DM) accounting for approximately 90-95\% of all cases [1]. The recent rapid increase in the prevalence of T2DM is in part due to an ageing population but may also be attributed to an increase in the number of overweight and obese people. It is estimated that approximately $6.6 \%$ of the world's population aged between 20 and 79 years will have diabetes in 2010, with this figure projected to increase to $7.8 \%$ by 2030 [2]

\section{KARGER}

Fax +4161306 1234 E-Mail karger@karger.ch www.karger.com
(C) 2010 S. Karger AG, Basel

0031-7012/10/0861-0044\$26.00/0

Accessible online at:

www.karger.com/pha
Dr. Walid Fakhoury

IMS Health

7 Harewood Avenue

London NW1 6JB (UK)

Tel. +44 203075 4818, Fax +44 203075 4840, E-Mail WFakhoury@uk.imshealth.com 
In the USA, the prevalence of T2DM among people over 20 years of age is $10.7 \%$ (23.5 million people) [3]. The total estimated cost of diabetes in the USA in 2007 was USD 174 billion, including USD 116 billion in excess medical expenditures and USD 58 billion in reduced national productivity [4]. In the UK, the prevalence of T2DM in 2009 within the adult population was 2.6 million, with the current estimated cost of treating diabetes and its related complications estimated to account for $10 \%$ of the National Health Service 2007/2008 budget or GBP 9 billion a year [5].

It has been suggested that ineffective drug therapies, poor patient adherence to therapy and inadequate management regimes may all contribute to the increasing incidence of complications linked to the disease [6]. Patients with T2DM are often required to undertake significant lifestyle and dietary changes in addition to prescription antidiabetes drug therapy. There exist a variety of antidiabetes drug therapies including metformin, sulfonylurea, thiazolidinediones, acarbose, prandial glucose regulators and insulin in various forms. A relatively new class of oral antihyperglycemic drugs has recently gained widespread use, namely the dipeptyl peptidase type 4 (DPP-4) inhibitors; these are best classified as incretin enhancers: sitagliptin (Januvia $^{\mathrm{TM}}$; Merck \& Co. Inc.) and vildagliptin (Galvus ${ }^{\mathrm{TM}}$; Novartis).

Sitagliptin $[7,8]$ is a once daily oral antihyperglycemic agent. It is used in addition to diet and exercise in the following ways:

- monotherapy in patients who are not satisfactorily controlled by diet and exercise and for whom metformin (an antidiabetic medicine) is contraindicated;

- in combination with metformin or a peroxisome proliferator-activated receptor (PPAR) $\gamma$ agonist such as thiazolidinediones, in patients who are not satisfactorily controlled on metformin or PPAR- $\gamma$ agonist used alone;

- in combination with a sulfonylurea in patients who are not adequately controlled with a sulfonylurea when used as monotherapy or for whom metformin is contraindicated;

- in combination with both metformin and a sulfonylurea or a PPAR- $\gamma$ agonist, in patients who are not satisfactorily controlled on the two medicines;

- in combination with insulin, with or without metformin, in patients who are not satisfactorily controlled by a stable dose of insulin $[7,8]$.

Sitagliptin is often prescribed at a dose of $100 \mathrm{mg}$ once daily and is not currently licensed for use in triple therapy [9].
Vildagliptin [10] is administered as dual oral therapy in combination with:

- metformin, in patients with insufficient glycemic control despite a maximal tolerated dose of monotherapy with metformin;

- a sulfonylurea, in patients with insufficient glycemic control despite a maximal tolerated dose of a sulfonylurea and for whom metformin is inappropriate due to contraindications or intolerance;

- a thiazolidinedione, in patients with insufficient glycemic control and for whom the use of a thiazolidinedione is appropriate;

- when used in dual combination with a sulfonylurea, the recommended dose of vildagliptin is $50 \mathrm{mg}$ once daily.

Another recently marketed class of antidiabetes therapies is the incretin mimetics, consisting of exenatide (Byetta $^{\mathrm{TM}}$; Eli Lilly \& Co.) and liraglutide (Victoza ${ }^{\mathrm{TM}}$; Novo Nordisk). Both are injectable medications.

Exenatide is administered in combination with metformin and/or sulfonylurea in patients who have not achieved adequate glycemic control by maximally tolerated doses of these oral antihyperglycemic drugs; it has also been recently approved as a monotherapy [11]. Exenatide therapy is initiated at a $5-\mu \mathrm{g}$ dose administered twice daily for at least 1 month in order to improve tolerability following which the dose can be increased to $10 \mu \mathrm{g}$ twice daily to further improve glycemic control [11].

Liraglutide (Victoza) is indicated for treatment in combination with:

- metformin or a sulfonylurea, in patients with insufficient glycemic control despite a maximal tolerated dose of monotherapy with metformin or sulfonylurea; - metformin and a sulfonylurea or metformin and a thiazolidinedione in patients with insufficient glycemic control despite dual therapy [12].

The starting dose of liraglutide is $0.6 \mathrm{mg}$ daily. After at least 1 week, the dose is increased to $1.2 \mathrm{mg}$, based on clinical response, and after at least 1 further week the dose can be increased to $1.8 \mathrm{mg}$ [12].

In order to provide an up-to-date comprehensive picture of the clinical efficacy and safety of the main types of incretin mimetics and DPP-4 inhibitors, we conducted a systematic review of the literature including trials of exenatide, liraglutide, sitagliptin and vildagliptin. Furthermore, a meta-analysis of the randomized controlled trials was performed, comparing each of these treatments to placebo. The aims were to: (1) describe the published evidence on the main clinical efficacy and safety outcomes of each of the treatments, and (2) identify a pooled 
estimate of the effect of each treatment versus placebo for the main outcomes so that comparative data are available in the literature for use in evidence-based practice and research.

\section{Materials and Methods}

The Embase, and Cochrane Library electronic databases were searched through Embase.com for English-language papers from January 2000 to July 2009, using the following search headings: type of diabetes, type of intervention, type of trial, comparators of interest and outcomes of interest. Clinical trials were included if they met the following criteria: (1) published in the English language, (2) primary study comparing either exenatide, liraglutide, vildagliptin or sitagliptin with placebo; (3) included patients with T2DM at least 18 years of age; (4) randomized controlled trial design (phase II, phase III, parallel design); (5) minimum study sample of 100 patients; (6) at least one baseline and posttreatment efficacy and/or safety outcome of interest (see Outcomes section below), and (7) reports of a dispersion measure [standard deviation, standard error of the mean or confidence interval (CI)], for both treatment arms of the study.

In addition, the reference lists and citation sections of recovered articles were searched for further studies, as were abstracts presented at the American Diabetes Association and the European Association Study of Diabetes conferences from August 2008 to July 2009.

The literature search identified 389 potentially relevant articles for screening. Of these, the research team (W.F., C.L., D.W.) excluded 312 articles (303 literature reviews and 9 conference abstracts). Any discrepancies were resolved by consensus between the 3 reviewers. Thus, 77 abstracts were retained for detailed evaluation. Of these, 40 were excluded for various reasons (less than 100 participants, no placebo group, no relevant outcome, no medication of interest), and 37 were retained for full data extraction for meta-analysis. One further study was identified by checking the reference lists of full-text articles, bringing the total to 38. For quality reasons, the team ensured (W.F., C.L., D.W.) that all selected studies were controlled clinical trials with proper randomization of patients and that they reported on all initially included patients according to the intention-to-treat principle and reported blinded outcome assessment.

Of the 38 trials identified, 8 were of exenatide, 7 of liraglutide, 12 of sitagliptin and 11 of vildagliptin (fig. 1).

\section{Outcomes}

The outcomes of interest in this analysis were: (1) change from baseline in hemoglobin $\mathrm{A}_{1 \mathrm{c}}\left(\mathrm{HbA}_{1 \mathrm{c}}\right)$; (2) change from baseline in weight, and (3) when available, body mass index. For these outcomes 1-3, the weighted mean differences (WMDs) and associated precision for the change from baseline were calculated in each study. Baseline and study endpoint $\mathrm{HbA}_{1}$, weight and patient-reported hypoglycemic events are presented in table 1.

\section{Data Extraction}

Data on the key characteristics of the study sample (e.g. age, gender, study duration, duration of diabetes, study location, sample size, definition of hypoglycemia by study), outcomes mea-

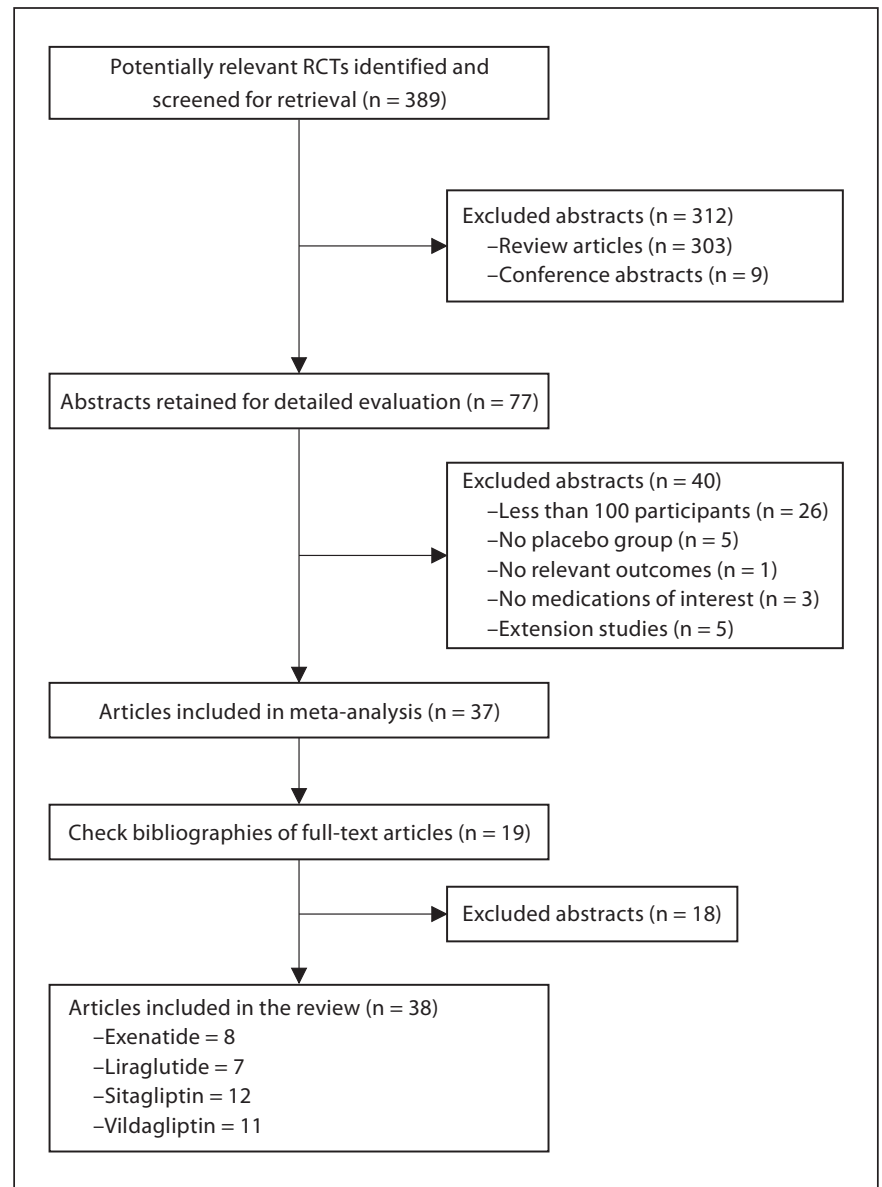

Fig. 1. Decision process for selecting relevant full-text articles for exenatide, liraglutide, sitagliptin and vildagliptin. $\mathrm{RCT}=$ Randomized controlled trial.

sured and results reported in each study were tabulated in a data extraction sheet. Data extraction was performed by one reviewer (D.W.), with the completed table checked for accuracy by a second reviewer (W.F.). The data extraction sheet was further reviewed by a statistician (C.L.) to ensure that all data needed for the metaanalysis were captured correctly.

\section{Statistical Analysis}

The statistical analysis was divided into 3 steps: (1) direct meta-analyses of each treatment versus placebo and (2) assessment of the impact of adjustment variables on the results of the metaanalyses. The outcome measures reported in each study were meta-analyzed to produce a pooled estimate of the effect of each treatment versus placebo for each outcome. Pooling was done using both fixed-effect and random-effect models. Heterogeneity was assessed using the $\chi^{2}$ test and the I2 statistic. If the I 2 statistics showed that significant heterogeneity existed between study results, a random-effect model was used, as advocated by Egger and Davey Smith [51]. 
Table 1. Baseline and study endpoint $\mathrm{HbA}_{\mathrm{lc}}(\%)$ and weight $(\mathrm{kg})$ values

\begin{tabular}{|c|c|c|c|c|c|c|c|c|}
\hline $\begin{array}{l}\text { Study } \\
\text { reference }\end{array}$ & Interventions & $\begin{array}{l}\text { Baseline } \\
\mathrm{HbA}_{1 \mathrm{c}}\end{array}$ & $\begin{array}{l}\mathrm{HbA}_{1 \mathrm{c}} \text { at } \\
\text { study } \\
\text { endpoint }\end{array}$ & $\begin{array}{l}\text { Change } \\
\text { in } \mathrm{HbA}_{1 \mathrm{c}}\end{array}$ & $\begin{array}{l}\text { Baseline } \\
\text { mean weight }\end{array}$ & $\begin{array}{l}\text { Weight at } \\
\text { study } \\
\text { endpoint }\end{array}$ & $\begin{array}{l}\text { Change } \\
\text { in weight }\end{array}$ & $\begin{array}{l}\text { Number of } \\
\text { hypoglyce- } \\
\text { mic episodes }\end{array}$ \\
\hline $\begin{array}{l}\text { Exenatide } \\
\text { Zinman } \\
\text { et al. [13], } \\
2007\end{array}$ & $\begin{array}{l}\text { rsus placebo } \\
\text { Exenatide } 10 \mu \mathrm{g} \text { twice daily } \\
\text { Placebo }\end{array}$ & $\begin{array}{l}7.9 \pm 0.9 \\
7.9 \pm 0.8\end{array}$ & $\begin{array}{l}\text { not } \\
\text { reported }\end{array}$ & $\begin{array}{r}0.09 \pm 0.10^{\mathrm{a}} \\
-0.89 \pm 0.09^{\mathrm{a}}\end{array}$ & $\begin{array}{l}97.5 \pm 18.8^{\mathrm{a}} \\
96.9 \pm 19.0^{\mathrm{a}}\end{array}$ & $\begin{array}{l}96.89 \pm 0.26^{\mathrm{a}} \\
95.38 \pm 0.25^{\mathrm{a}}\end{array}$ & $\begin{array}{l}-1.75 \pm 0.25^{\mathrm{a}} \\
-0.24 \pm 0.26^{\mathrm{a}}\end{array}$ & 13 \\
\hline $\begin{array}{l}\text { De Fronzo } \\
\text { et al. [14], } \\
2005\end{array}$ & $\begin{array}{l}\text { Exenatide } 5 \mu \mathrm{g} \text { twice daily } \\
\text { Exenatide } 10 \mu \mathrm{g} \text { twice daily } \\
\text { Placebo twice daily }\end{array}$ & $\begin{array}{l}8.3 \pm 1.1 \\
8.2 \pm 1.0 \\
8.2 \pm 1.0\end{array}$ & $\begin{array}{l}\text { not } \\
\text { reported }\end{array}$ & $\begin{array}{r}-0.4 \pm 0.1^{\mathrm{a}} \\
-0.8 \pm 0.1^{\mathrm{a}} \\
0.1 \pm 0.1^{\mathrm{a}}\end{array}$ & $\begin{array}{l}100 \pm 22 \\
101 \pm 20 \\
100 \pm 19\end{array}$ & $\begin{array}{l}\text { not } \\
\text { reported }\end{array}$ & $\begin{array}{l}-1.6 \pm 0.4^{\mathrm{a}} \\
-2.8 \pm 0.5^{\mathrm{a}} \\
-0.3 \pm 0.3^{\mathrm{a}}\end{array}$ & 11 \\
\hline $\begin{array}{l}\text { Gao } \\
\text { et al. [15], } \\
2009\end{array}$ & $\begin{array}{l}\text { Exenatide } 10 \mu \mathrm{g} \text { twice daily } \\
\text { Placebo twice daily }\end{array}$ & $\begin{array}{l}8.3 \pm 1.0 \\
8.3 \pm 1.0\end{array}$ & $\begin{array}{l}\text { not } \\
\text { reported }\end{array}$ & $\begin{array}{l}-1.2 \\
-0.4\end{array}$ & $\begin{array}{l}69.6 \pm 11.2 \\
67.9 \pm 11.1\end{array}$ & $\begin{array}{l}\text { not } \\
\text { reported }\end{array}$ & $\begin{array}{l}-1.2 \pm 2.34 \\
-0.1 \pm 1.94\end{array}$ & 41 \\
\hline $\begin{array}{l}\text { Kendall } \\
\text { et al. [16], } \\
2005\end{array}$ & $\begin{array}{l}\text { Exenatide } 5 \mu \mathrm{g} \text { twice daily } \\
\text { Exenatide } 10 \mu \mathrm{g} \text { twice daily } \\
\text { Placebo }\end{array}$ & $\begin{array}{l}8.5 \pm 1.0 \\
8.5 \pm 1.1 \\
8.5 \pm 1.0\end{array}$ & $\begin{array}{l}\text { not } \\
\text { reported }\end{array}$ & $\begin{array}{r}-0.55 \pm 0.07^{\mathrm{a}} \\
-0.77 \pm 0.08^{\mathrm{a}} \\
0.23 \pm 0.07^{\mathrm{a}}\end{array}$ & $\begin{array}{l}97 \pm 19 \\
98 \pm 21 \\
99 \pm 19\end{array}$ & $\begin{array}{l}\text { not } \\
\text { reported }\end{array}$ & $\begin{array}{l}-1.6 \pm 0.2^{\mathrm{a}} \\
-1.6 \pm 0.2^{\mathrm{a}} \\
-0.9 \pm 0.2^{\mathrm{a}}\end{array}$ & 114 \\
\hline $\begin{array}{l}\text { Moretto } \\
\text { et al. [17], } \\
2008\end{array}$ & $\begin{array}{l}\text { Exenatide } 5 \mu \mathrm{g} \text { twice daily } \\
\text { Exenatide } 10 \mu \mathrm{g} \text { twice daily } \\
\text { Placebo twice daily }\end{array}$ & $\begin{array}{l}7.9 \pm 1.0 \\
7.8 \pm 1.0 \\
7.8 \pm 0.9\end{array}$ & $\begin{array}{l}\text { not } \\
\text { reported }\end{array}$ & $\begin{array}{l}-0.7 \pm 0.1^{\mathrm{a}} \\
-0.9 \pm 0.1^{\mathrm{a}} \\
-0.2 \pm 0.1^{\mathrm{a}}\end{array}$ & $\begin{array}{l}85 \pm 15 \\
86 \pm 16 \\
86 \pm 16\end{array}$ & $\begin{array}{l}\text { not } \\
\text { reported }\end{array}$ & $\begin{array}{l}-2.8 \pm 0.3^{\mathrm{a}} \\
-3.1 \pm 0.3^{\mathrm{a}} \\
-1.4 \pm 0.3^{\mathrm{a}}\end{array}$ & 7 \\
\hline $\begin{array}{l}\text { Poon } \\
\text { et al. [18], } \\
2005\end{array}$ & $\begin{array}{l}\text { Exenatide } 2.5 \mu \mathrm{g} \text { twice daily } \\
\text { Exenatide } 5 \mu \mathrm{g} \text { twice daily } \\
\text { Exenatide } 7.5 \mu \mathrm{g} \text { twice daily } \\
\text { Exenatide } 10 \mu \mathrm{g} \text { twice daily } \\
\text { Placebo twice daily }\end{array}$ & $\begin{array}{l}7.7 \pm 0.7 \\
7.5 \pm 0.7 \\
7.4 \pm 0.7 \\
7.5 \pm 0.5 \\
7.5 \pm 0.8\end{array}$ & $\begin{array}{l}\text { not } \\
\text { reported }\end{array}$ & $\begin{array}{r}-0.3 \pm 0.1^{\mathrm{a}} \\
-0.4 \pm 0.1^{\mathrm{a}} \\
-0.5 \pm 0.0^{\mathrm{a}} \\
-0.5 \pm 0.1^{\mathrm{a}} \\
0.1 \pm 0.1^{\mathrm{a}}\end{array}$ & $\begin{array}{r}98.6 \pm 22.6 \\
103.6 \pm 15.4 \\
99.5 \pm 21.0 \\
92.4 \pm 15.1 \\
102.6 \pm 19.9\end{array}$ & $\begin{array}{l}\text { not } \\
\text { reported }\end{array}$ & $\begin{array}{r}-0.07 \pm 0.3^{\mathrm{a}} \\
-0.7 \pm 0.2^{\mathrm{a}} \\
-1.4 \pm 0.3^{\mathrm{a}} \\
-1.8 \pm 0.3^{\mathrm{a}} \\
0.0 \pm 0.3^{\mathrm{a}}\end{array}$ & 2 \\
\hline $\begin{array}{l}\text { Buse } \\
\text { et al. [19], } \\
2004\end{array}$ & $\begin{array}{l}\text { Exenatide } 5 \mu \mathrm{g} \text { b.i.d. } \\
\text { Exenatide } 10 \mu \mathrm{g} \text { b.i.d. } \\
\text { Placebo b.i.d. }\end{array}$ & $\begin{array}{l}8.5 \pm 1.1 \\
8.6 \pm 1.2 \\
8.7 \pm 1.2\end{array}$ & $\begin{array}{l}\text { not } \\
\text { reported }\end{array}$ & $\begin{array}{r}-0.46 \pm 0.12^{\mathrm{a}} \\
-0.86 \pm 0.11^{\mathrm{a}} \\
0.12 \pm 0.09^{\mathrm{a}}\end{array}$ & $\begin{array}{l}94.9 \pm 1.9^{\mathrm{a}} \\
95.2 \pm 1.6^{\mathrm{a}} \\
99.1 \pm 1.7^{\mathrm{a}}\end{array}$ & $\begin{array}{l}\text { not } \\
\text { reported }\end{array}$ & $\begin{array}{l}-0.9 \pm 0.3^{\mathrm{a}} \\
-1.6 \pm 0.3^{\mathrm{a}} \\
-0.6 \pm 0.3^{\mathrm{a}}\end{array}$ & 64 \\
\hline $\begin{array}{l}\text { Fineman } \\
\text { et al. [20], } \\
2003\end{array}$ & $\begin{array}{l}\text { Exenatide } 0.08 \mu \mathrm{g} \text { (breakfast, dinner, } \\
\text { bedtime) } \\
\text { Exenatide } 0.08 \mu \mathrm{g} \text { (breakfast, dinner) } \\
\text { Exenatide } 0.08 \mu \mathrm{g} \text { (breakfast, bedtime) } \\
\text { Placebo } 0.08 \mu \mathrm{g} \text { (breakfast, dinner, } \\
\text { bedtime) }\end{array}$ & $\begin{array}{l}9.1 \pm 1.2 \\
9.3 \pm 1.0 \\
9.2 \pm 1.1 \\
9.4 \pm 1.3\end{array}$ & $\begin{array}{l}\text { not } \\
\text { reported }\end{array}$ & $\begin{array}{l}-1.1 \pm 0.07^{\mathrm{a}} \\
-0.71 \pm 0.12^{\mathrm{a}} \\
-1.02 \pm 0.11^{\mathrm{a}} \\
-0.3 \pm 0.11^{\mathrm{a}}\end{array}$ & $\begin{array}{l}97.0 \pm 16.7 \\
98.2 \pm 16.0 \\
96.8 \pm 17.5 \\
97.6 \pm 16.7\end{array}$ & $\begin{array}{l}\text { not } \\
\text { reported }\end{array}$ & $\begin{array}{l}\text { not } \\
\text { reported }\end{array}$ & $\begin{array}{l}\text { not } \\
\text { reported }\end{array}$ \\
\hline $\begin{array}{l}\text { Liraglutide } \\
\text { Zinman } \\
\text { et al. [21], } \\
2009\end{array}$ & $\begin{array}{l}\text { ersus placebo } \\
\text { Liraglutide } 1.2 \mathrm{mg} \text { once daily } \\
\text { Liraglutide } 1.8 \mathrm{mg} \text { once daily } \\
\text { Placebo once daily }\end{array}$ & $\begin{array}{l}8.5 \pm 1.2 \\
8.6 \pm 1.2 \\
8.4 \pm 1.2\end{array}$ & $\begin{array}{l}7.0 \\
7.1 \\
7.9\end{array}$ & $\begin{array}{l}-1.5 \pm 0.1^{\mathrm{a}} \\
-1.5 \pm 0.1^{\mathrm{a}} \\
-0.5 \pm 0.1^{\mathrm{a}}\end{array}$ & $\begin{array}{l}\text { not } \\
\text { reported }\end{array}$ & $\begin{array}{l}\text { not } \\
\text { reported }\end{array}$ & $\begin{array}{l}-1.75 \pm 2.75 \\
-0.24 \pm 2.75\end{array}$ & \\
\hline $\begin{array}{l}\text { Madsbad } \\
\text { et al. [22], } \\
2004\end{array}$ & $\begin{array}{l}\text { Liraglutide } 0.045 \mathrm{mg} \text { once daily } \\
\text { Liraglutide } 0.225 \mathrm{mg} \text { once daily } \\
\text { Liraglutide } 0.45 \mathrm{mg} \text { once daily } \\
\text { Liraglutide } 0.60 \mathrm{mg} \text { once daily } \\
\text { Liraglutide } 0.75 \mathrm{mg} \text { once daily } \\
\text { Placebo once daily }\end{array}$ & $\begin{array}{l}7.4 \pm 0.8 \\
7.9 \pm 0.8 \\
7.7 \pm 1.0 \\
7.4 \pm 1.2 \\
7.4 \pm 0.9 \\
7.4 \pm 1.2\end{array}$ & $\begin{array}{l}7.91 \\
7.51 \\
7.31 \\
6.81 \\
6.81 \\
7.31\end{array}$ & $\begin{array}{l}\text { not } \\
\text { reported }\end{array}$ & $\begin{array}{l}\text { not } \\
\text { reported }\end{array}$ & $\begin{array}{l}\text { not } \\
\text { reported }\end{array}$ & $\begin{array}{l}\text { not } \\
\text { reported }\end{array}$ & 1 \\
\hline $\begin{array}{l}\text { Marre } \\
\text { et al. [23], } \\
2009\end{array}$ & $\begin{array}{l}\text { Liraglutide } 0.6 \mathrm{mg} \text { once daily } \\
\text { Liraglutide } 1.2 \mathrm{mg} \text { once daily } \\
\text { Liraglutide } 1.8 \mathrm{mg} \text { once daily } \\
\text { Placebo once daily }\end{array}$ & $\begin{array}{l}8.4 \pm 1.0 \\
8.5 \pm 1.1 \\
8.5 \pm 0.9 \\
8.4 \pm 1.0\end{array}$ & $\begin{array}{l}7.8 \pm 0.15^{\mathrm{a}} \\
7.4 \pm 0.16^{\mathrm{a}} \\
7.4 \pm 0.16^{\mathrm{a}} \\
8.6 \pm 0.24^{\mathrm{a}}\end{array}$ & $\begin{array}{r}-0.6 \pm 0.15^{\mathrm{a}} \\
-1.08 \pm 0.11^{\mathrm{a}} \\
-1.13 \pm 0.17^{\mathrm{a}} \\
0.23 \pm 0.16^{\mathrm{a}}\end{array}$ & $\begin{array}{l}82.6 \pm 17.7 \\
83.0 \pm 18.1 \\
81.9 \pm 17.1 \\
80.6 \pm 17.0\end{array}$ & $\begin{array}{l}\text { not } \\
\text { reported }\end{array}$ & $\begin{array}{r}0.7 \pm 0.4^{\mathrm{a}} \\
0.3 \pm 0.3^{\mathrm{a}} \\
-0.2 \pm 0.4^{\mathrm{a}} \\
-0.1 \pm 0.5^{\mathrm{a}}\end{array}$ & 52 \\
\hline $\begin{array}{l}\text { Nauck } \\
\text { et al. [24], } \\
2009\end{array}$ & $\begin{array}{l}\text { Liraglutide } 0.6 \mathrm{mg} \text { once daily } \\
\text { Liraglutide } 1.2 \mathrm{mg} \text { once daily } \\
\text { Liraglutide } 1.8 \mathrm{mg} \text { once daily } \\
\text { Placebo once daily }\end{array}$ & $\begin{array}{l}8.4 \pm 0.9 \\
8.3 \pm 1.0 \\
8.4 \pm 1.0 \\
8.4 \pm 1.1\end{array}$ & $\begin{array}{l}\text { not } \\
\text { reported }\end{array}$ & $\begin{array}{r}-0.7 \pm 0.1^{\mathrm{a}} \\
-1.0 \pm 0.1^{\mathrm{a}} \\
-1.0 \pm 0.1^{\mathrm{a}} \\
0.1 \pm 0.1^{\mathrm{a}}\end{array}$ & $\begin{array}{l}\text { not } \\
\text { reported }\end{array}$ & $\begin{array}{l}\text { not } \\
\text { reported }\end{array}$ & $\begin{array}{l}\text { not } \\
\text { reported }\end{array}$ & 21 \\
\hline $\begin{array}{l}\text { Seino } \\
\text { et al. [25], } \\
2008\end{array}$ & $\begin{array}{l}\text { Liraglutide } 0.1 \mathrm{mg} \text { once daily } \\
\text { Liraglutide } 0.3 \mathrm{mg} \text { once daily } \\
\text { Liraglutide } 0.6 \mathrm{mg} \text { once daily } \\
\text { Liraglutide } 0.9 \mathrm{mg} \text { once daily } \\
\text { Placebo once daily }\end{array}$ & $\begin{array}{l}8.50 \pm 0.84 \\
8.24 \pm 0.92 \\
8.21 \pm 0.83 \\
8.12 \pm 0.98 \\
8.43 \pm 1.02\end{array}$ & $\begin{array}{l}7.78 \pm 0.91 \\
7.17 \pm 1.01 \\
6.71 \pm 0.92 \\
6.45 \pm 0.77 \\
8.52 \pm 1.23\end{array}$ & $\begin{array}{l}\text { not } \\
\text { reported }\end{array}$ & $\begin{array}{l}64.26 \pm 10.46 \\
61.52 \pm 9.46 \\
61.54 \pm 10.98 \\
61.48 \pm 10.55 \\
62.00 \pm 10.97\end{array}$ & $\begin{array}{l}64.21 \pm 10.67 \\
61.67 \pm 11.39 \\
61.42 \pm 9.68 \\
61.00 \pm 11.07 \\
61.05 \pm 10.89\end{array}$ & $\begin{array}{l}\text { not } \\
\text { reported }\end{array}$ & 0 \\
\hline
\end{tabular}


Table 1 (continued)

\begin{tabular}{|c|c|c|c|c|c|c|c|c|}
\hline $\begin{array}{l}\text { Study } \\
\text { reference }\end{array}$ & Interventions & $\begin{array}{l}\text { Baseline } \\
\mathrm{HbA}_{1 \mathrm{c}}\end{array}$ & $\begin{array}{l}\mathrm{HbA}_{1 \mathrm{c}} \text { at } \\
\text { study } \\
\text { endpoint }\end{array}$ & $\begin{array}{l}\text { Change } \\
\text { in } \mathrm{HbA}_{1 \mathrm{c}}\end{array}$ & $\begin{array}{l}\text { Baseline } \\
\text { mean weight }\end{array}$ & $\begin{array}{l}\text { Weight at } \\
\text { study } \\
\text { endpoint }\end{array}$ & $\begin{array}{l}\text { Change } \\
\text { in weight }\end{array}$ & $\begin{array}{l}\text { Number of } \\
\text { hypoglyce- } \\
\text { mic episodes }\end{array}$ \\
\hline $\begin{array}{l}\text { Vilsboll } \\
\text { et al. [26], } \\
2007\end{array}$ & $\begin{array}{l}\text { Liraglutide } 0.65 \mathrm{mg} \text { once daily } \\
\text { Liraglutide } 1.25 \mathrm{mg} \text { once daily } \\
\text { Liraglutide } 1.90 \mathrm{mg} \text { once daily } \\
\text { Placebo once daily }\end{array}$ & $\begin{array}{l}8.1 \pm 0.6 \\
8.3 \pm 0.8 \\
8.5 \pm 0.9 \\
8.2 \pm 0.7\end{array}$ & $\begin{array}{l}\text { not } \\
\text { reported }\end{array}$ & $\begin{array}{c}-0.98 \\
-1.4 \\
-1.45 \\
0.29\end{array}$ & $\begin{array}{l}\text { not } \\
\text { reported }\end{array}$ & $\begin{array}{l}\text { not } \\
\text { reported }\end{array}$ & $\begin{array}{l}\text { not } \\
\text { reported }\end{array}$ & 0 \\
\hline $\begin{array}{l}\text { Russell- } \\
\text { Jones et al. } \\
\text { [27], 2008 } \\
\text { (abstract } \\
\text { only) }\end{array}$ & $\begin{array}{l}\text { Liraglutide } 1.8 \mathrm{mg} \text { once daily } \\
\text { Placebo twice daily }\end{array}$ & $\begin{array}{l}8.3 \\
8.2\end{array}$ & $\begin{array}{l}7.0 \pm 1.0 \\
8.1 \pm 1.3\end{array}$ & $\begin{array}{l}-1.3 \pm 0.09^{\mathrm{a}} \\
-0.2 \pm 0.11^{\mathrm{a}}\end{array}$ & $\begin{array}{l}\text { not } \\
\text { reported }\end{array}$ & $\begin{array}{l}\text { not } \\
\text { reported }\end{array}$ & $\begin{array}{l}-1.80 \pm 5.03 \\
-0.42 \pm 4.18\end{array}$ & $\begin{array}{l}\text { not } \\
\text { reported }\end{array}$ \\
\hline $\begin{array}{l}\text { Sitagliptin ve } \\
\text { Scott } \\
\text { et al. [28], } \\
2008\end{array}$ & $\begin{array}{l}\text { rsus placebo } \\
\text { Sitagliptin } 100 \text { mg once daily } \\
\text { Placebo once daily }\end{array}$ & $\begin{array}{l}7.8 \pm 1.0 \\
7.7 \pm 0.9\end{array}$ & $\begin{array}{l}7.01 \pm 0.86 \\
7.47 \pm 1.05\end{array}$ & $\begin{array}{l}-0.73 \\
-0.22\end{array}$ & $\begin{array}{l}83.1 \pm 17.1 \\
84.6 \pm 16.5\end{array}$ & $\begin{array}{l}\text { not } \\
\text { reported }\end{array}$ & $\begin{array}{l}-0.4 \pm 1.98 \\
-0.8 \pm 1.96\end{array}$ & 12 \\
\hline $\begin{array}{l}\text { Aschner } \\
\text { et al. [29], } \\
2006\end{array}$ & $\begin{array}{l}\text { Sitagliptin } 100 \mathrm{mg} \text { once daily } \\
\text { Sitagliptin } 200 \mathrm{mg} \text { once daily } \\
\text { Placebo once daily }\end{array}$ & $\begin{array}{l}8.01 \pm 0.88 \\
8.08 \pm 0.94 \\
8.03 \pm 0.82\end{array}$ & $\begin{array}{l}7.39 \pm 1.15 \\
7.31 \pm 1.14 \\
8.20 \pm 1.37\end{array}$ & $\begin{array}{r}-0.61 \\
-0.76 \\
0.18\end{array}$ & $\begin{array}{l}85.0 \pm 18 \\
83.7 \pm 19.2 \\
85.0 \pm 18.1\end{array}$ & $\begin{array}{l}\text { not } \\
\text { reported }\end{array}$ & $\begin{array}{l}-0.2 \pm 0.2^{\mathrm{a}} \\
-0.1 \pm 0.2^{\mathrm{a}} \\
-1.1 \pm 0.2^{\mathrm{a}}\end{array}$ & 5 \\
\hline $\begin{array}{l}\text { Charbonnel } \\
\text { et al. [30], } \\
2006\end{array}$ & $\begin{array}{l}\text { Sitagliptin } 100 \text { mg once daily } \\
\text { Placebo once daily }\end{array}$ & $\begin{array}{l}7.96 \pm 0.81 \\
8.03 \pm 0.82\end{array}$ & $\begin{array}{l}7.26 \pm 0.97 \\
7.95 \pm 1.10\end{array}$ & $\begin{array}{l}-0.67 \\
-0.02\end{array}$ & $\begin{array}{l}86.7 \pm 17.8 \\
89.6 \pm 17.5\end{array}$ & $\begin{array}{l}\text { not } \\
\text { reported }\end{array}$ & $\begin{array}{l}\text { not } \\
\text { reported }\end{array}$ & 6 \\
\hline $\begin{array}{l}\text { Goldstein } \\
\text { et al. [31], } \\
2007\end{array}$ & $\begin{array}{l}\text { Sitagliptin } 50 \mathrm{mg} \text { twice daily } \\
\text { Sitagliptin } 50 \mathrm{mg} \text { twice daily } \\
\text { Sitagliptin } 100 \mathrm{mg} \text { once daily } \\
\text { Placebo }\end{array}$ & $\begin{array}{l}8.79 \pm 1.00 \\
8.76 \pm 0.95 \\
8.87 \pm 0.99 \\
8.68 \pm 1.00\end{array}$ & $\begin{array}{l}7.37 \pm 1.20 \\
6.87 \pm 1.09 \\
8.18 \pm 1.45 \\
8.88 \pm 1.47\end{array}$ & $\begin{array}{r}-1.4 \\
-1.9 \\
-0.66 \\
0.17\end{array}$ & $\begin{array}{l}\text { not } \\
\text { reported }\end{array}$ & $\begin{array}{l}\text { not } \\
\text { reported }\end{array}$ & $\begin{array}{l}\text { not } \\
\text { reported }\end{array}$ & 7 \\
\hline $\begin{array}{l}\text { Hanefeld } \\
\text { et al. [32], } \\
2007\end{array}$ & $\begin{array}{l}\text { Sitagliptin } 25 \mathrm{mg} \text { once daily } \\
\text { Sitagliptin } 50 \mathrm{mg} \text { once daily } \\
\text { Sitagliptin } 100 \mathrm{mg} \text { once daily } \\
\text { Sitagliptin } 50 \mathrm{mg} \text { twice daily } \\
\text { Placebo }\end{array}$ & $\begin{array}{l}7.71 \pm 0.91 \\
7.60 \pm 0.94 \\
7.78 \pm 0.90 \\
7.79 \pm 0.85 \\
7.59 \pm 0.89\end{array}$ & $\begin{array}{l}7.47 \pm 1.30 \\
7.22 \pm 1.02 \\
7.38 \pm 1.11 \\
7.41 \pm 1.10 \\
7.76 \pm 1.11\end{array}$ & $\begin{array}{r}-0.28 \\
-0.44 \\
-0.44 \\
-0.43 \\
0.12\end{array}$ & $\begin{array}{l}\text { not } \\
\text { reported }\end{array}$ & $\begin{array}{l}\text { not } \\
\text { reported }\end{array}$ & $\begin{array}{l}\text { not } \\
\text { reported }\end{array}$ & 5 \\
\hline $\begin{array}{l}\text { Hermansen } \\
\text { et al. [33], } \\
2007\end{array}$ & $\begin{array}{l}\text { Sitagliptin } 100 \mathrm{mg} \text { once daily } \\
\text { Sitagliptin } 100 \mathrm{mg} \text { once daily } \\
\text { Sitagliptin cohort } \\
\text { Placebo once daily } \\
\text { Placebo once daily } \\
\text { Placebo cohort }\end{array}$ & $\begin{array}{l}8.42 \pm 0.79 \\
8.27 \pm 0.73 \\
8.34 \pm 0.76 \\
8.43 \pm 0.80 \\
8.26 \pm 0.68 \\
8.34 \pm 0.74\end{array}$ & $\begin{array}{l}\text { not } \\
\text { reported }\end{array}$ & $\begin{array}{l}-0.3 \\
-0.59 \\
-0.45 \\
0.27 \\
0.3 \\
0.28\end{array}$ & $\begin{array}{l}85.8 \pm 22.5 \\
87.2 \pm 19.7 \\
86.5 \pm 21.1 \\
85.1 \pm 22.6 \\
86.7 \pm 21.1 \\
85.9 \pm 21.8\end{array}$ & $\begin{array}{l}\text { not } \\
\text { reported }\end{array}$ & $\begin{array}{l}1.1 \\
\text { not reported } \\
0.8 \\
\text { not reported } \\
\text { not reported } \\
\text { not reported }\end{array}$ & $\begin{array}{l}27 \text { (sitagliptin } \\
100 \text { mg once } \\
\text { daily) } \\
8 \text { (sitagliptin } \\
100 \mathrm{mg} \text { once } \\
\text { daily) } \\
19 \text { (sitagliptin } \\
\text { cohort) }\end{array}$ \\
\hline $\begin{array}{l}\text { Mohan } \\
\text { et al. [34], } \\
2009\end{array}$ & $\begin{array}{l}\text { Sitagliptin } 100 \mathrm{mg} \text { once daily } \\
\text { Placebo once daily }\end{array}$ & $\begin{array}{l}8.7 \pm 1.0 \\
8.7 \pm 1.0\end{array}$ & $\begin{array}{l}8.0 \pm 1.3 \\
9.1 \pm 1.6\end{array}$ & $\begin{array}{r}-0.7 \\
0.3\end{array}$ & $\begin{array}{l}66.8 \pm 10.2 \\
66.6 \pm 11.4\end{array}$ & & $\begin{array}{l}0.6 \pm 0.1 \\
0.0 \pm 0.2\end{array}$ & 0 \\
\hline $\begin{array}{l}\text { Nonaka } \\
\text { et al. [35], } \\
2008\end{array}$ & $\begin{array}{l}\text { Sitagliptin } 100 \text { mg once daily } \\
\text { Placebo once daily }\end{array}$ & $\begin{array}{l}7.54 \pm 0.85 \\
7.69 \pm 0.86\end{array}$ & $\begin{array}{l}6.90 \pm 1.00 \\
8.09 \pm 1.04\end{array}$ & $\begin{array}{r}-0.65 \\
0.41\end{array}$ & $\begin{array}{l}\text { not } \\
\text { reported }\end{array}$ & $\begin{array}{l}\text { not } \\
\text { reported }\end{array}$ & $\begin{array}{l}-0.1 \pm 1.56 \\
-0.7 \pm 1.33\end{array}$ & 0 \\
\hline $\begin{array}{l}\text { Raz } \\
\text { et al. [36], } \\
2008\end{array}$ & $\begin{array}{l}\text { Sitagliptin } 100 \mathrm{mg} \text { once daily } \\
\text { Placebo once daily }\end{array}$ & $\begin{array}{l}9.3 \pm 0.9 \\
9.1 \pm 0.8\end{array}$ & $\begin{array}{l}\text { not } \\
\text { reported }\end{array}$ & $\begin{array}{r}-1.0 \\
0.0\end{array}$ & $\begin{array}{l}81.5 \pm 16.8 \\
81.2 \pm 19.4\end{array}$ & $\begin{array}{l}\text { not } \\
\text { reported }\end{array}$ & $\begin{array}{l}\text { not } \\
\text { reported }\end{array}$ & 5 \\
\hline $\begin{array}{l}\text { Scott } \\
\text { et al. [37], } \\
2007\end{array}$ & $\begin{array}{l}\text { Sitagliptin } 5 \mathrm{mg} \text { twice daily } \\
\text { Sitagliptin } 12.5 \mathrm{mg} \text { twice daily } \\
\text { Sitagliptin } 25 \mathrm{mg} \text { twice daily } \\
\text { Sitagliptin } 50 \mathrm{mg} \text { twice daily } \\
\text { Placebo }\end{array}$ & $\begin{array}{l}7.89 \pm 0.94 \\
7.85 \pm 0.88 \\
7.89 \pm 0.94 \\
7.83 \pm 0.95 \\
7.88 \pm 0.96\end{array}$ & $\begin{array}{l}7.77 \pm 1.22 \\
7.48 \pm 0.98 \\
7.50 \pm 1.14 \\
7.34 \pm 1.01 \\
8.14 \pm 1.23\end{array}$ & $\begin{array}{r}-0.15 \\
-0.41 \\
-0.43 \\
-0.54 \\
0.23\end{array}$ & $\begin{array}{l}\text { not } \\
\text { reported }\end{array}$ & $\begin{array}{l}\text { not } \\
\text { reported }\end{array}$ & $\begin{array}{l}\text { not } \\
\text { reported }\end{array}$ & 2 \\
\hline
\end{tabular}


Table 1 (continued)

\begin{tabular}{|c|c|c|c|c|c|c|c|c|}
\hline $\begin{array}{l}\text { Study } \\
\text { reference }\end{array}$ & Interventions & $\begin{array}{l}\text { Baseline } \\
\mathrm{HbA}_{1 \mathrm{c}}\end{array}$ & $\begin{array}{l}\mathrm{HbA}_{1 \mathrm{c}} \text { at } \\
\text { study } \\
\text { endpoint }\end{array}$ & $\begin{array}{l}\text { Change } \\
\text { in } \mathrm{HbA}_{1 \mathrm{c}}\end{array}$ & $\begin{array}{l}\text { Baseline } \\
\text { mean weight }\end{array}$ & $\begin{array}{l}\text { Weight at } \\
\text { study } \\
\text { endpoint }\end{array}$ & $\begin{array}{l}\text { Change } \\
\text { in weight }\end{array}$ & $\begin{array}{l}\text { Number of } \\
\text { hypoglyce- } \\
\text { mic episodes }\end{array}$ \\
\hline $\begin{array}{l}\text { Rosenstock } \\
\text { et al. [38], } \\
2006\end{array}$ & $\begin{array}{l}\text { Sitagliptin } 5 \mathrm{mg} \text { once daily } \\
\text { Placebo }\end{array}$ & $\begin{array}{l}8.05 \pm 0.81 \\
8.00 \pm 0.83\end{array}$ & $\begin{array}{l}7.17 \pm 0.91 \\
7.82 \pm 1.10\end{array}$ & $\begin{array}{l}-0.85 \\
-0.15\end{array}$ & $\begin{array}{l}90.9 \pm 17.0 \\
86.4 \pm 17.4\end{array}$ & $\begin{array}{l}\text { not } \\
\text { reported }\end{array}$ & $\begin{array}{l}1.8 \\
1.5\end{array}$ & 2 \\
\hline $\begin{array}{l}\text { Raz } \\
\text { et al. [39], } \\
2006\end{array}$ & $\begin{array}{l}\text { Sitagliptin } 100 \mathrm{mg} \text { once daily } \\
\text { Sitagliptin } 200 \mathrm{mg} \text { once daily } \\
\text { Placebo }\end{array}$ & $\begin{array}{l}8.04 \pm 0.82 \\
8.14 \pm 0.91 \\
8.05 \pm 0.90\end{array}$ & $\begin{array}{l}7.58 \pm 1.35 \\
7.58 \pm 1.35 \\
8.21 \pm 1.35\end{array}$ & $\begin{array}{r}-0.48 \\
-0.36 \\
0.12\end{array}$ & $\begin{array}{l}89.7 \pm 19.1 \\
89.6 \pm 19.4 \\
92.8 \pm 18.8\end{array}$ & $\begin{array}{l}\text { not } \\
\text { reported }\end{array}$ & $\begin{array}{l}\text { not } \\
\text { reported }\end{array}$ & 12 \\
\hline \multicolumn{9}{|c|}{ Vildagliptin versus placebo } \\
\hline $\begin{array}{l}\text { Ahren } \\
\text { et al. [40], } \\
2004\end{array}$ & $\begin{array}{l}\text { Vildagliptin } 50 \mathrm{mg} \text { once daily } \\
\text { Placebo once daily }\end{array}$ & $\begin{array}{l}7.7 \pm 0.1^{\mathrm{a}} \\
7.9 \pm 0.1^{\mathrm{a}}\end{array}$ & $\begin{array}{l}\text { not } \\
\text { reported }\end{array}$ & $\begin{array}{r}-0.6 \pm 0.1 \\
0.1 \pm 0.1\end{array}$ & $\begin{array}{l}\text { not } \\
\text { reported }\end{array}$ & $\begin{array}{l}\text { not } \\
\text { reported }\end{array}$ & & 3 \\
\hline $\begin{array}{l}\text { Ristic } \\
\text { et al. [41], } \\
2005\end{array}$ & $\begin{array}{l}\text { Vildagliptin } 25 \mathrm{mg} \text { once daily } \\
\text { Vildagliptin } 25 \mathrm{mg} \text { twice daily } \\
\text { Vildagliptin } 50 \mathrm{mg} \text { once daily } \\
\text { Vildagliptin } 100 \mathrm{mg} \text { once daily } \\
\text { Placebo once daily }\end{array}$ & $\begin{array}{l}7.64 \pm 0.69 \\
7.73 \pm 0.80 \\
7.70 \pm 0.82 \\
7.64 \pm 0.75 \\
7.76 \pm 0.83\end{array}$ & $\begin{array}{l}\text { not } \\
\text { reported }\end{array}$ & $\begin{array}{l}-0.31 \pm 0.11 \\
-0.27 \pm 0.10 \\
-0.56 \pm 0.10 \\
-0.53 \pm 0.10 \\
-0.13 \pm 0.10\end{array}$ & $\begin{array}{l}89.4 \pm 2.8^{\mathrm{a}} \\
91.1 \pm 2.0^{\mathrm{a}} \\
87.9 \pm 2.3^{\mathrm{a}} \\
91.5 \pm 2.2^{\mathrm{a}} \\
92.0 \pm 2.1^{\mathrm{a}}\end{array}$ & $\begin{array}{l}\text { not } \\
\text { reported }\end{array}$ & $\begin{array}{r}0.06 \pm 0.33^{\mathrm{a}} \\
-0.55 \pm 0.32^{\mathrm{a}} \\
0.04 \pm 0.33^{\mathrm{a}} \\
-0.07 \pm 0.31^{\mathrm{a}} \\
-0.73 \pm 0.33^{\mathrm{a}}\end{array}$ & 14 \\
\hline $\begin{array}{l}\text { Bosi } \\
\text { et al. [42], } \\
2007\end{array}$ & $\begin{array}{l}\text { Vildagliptin } 50 \text { mg once daily } \\
\text { Vildagliptin } 100 \mathrm{mg} \text { once daily } \\
\text { Placebo once daily }\end{array}$ & $\begin{array}{l}8.4 \pm 0.9 \\
8.4 \pm 1.0 \\
8.3 \pm 0.9\end{array}$ & $\begin{array}{l}\text { not } \\
\text { reported }\end{array}$ & $\begin{array}{r}-0.9 \pm 0.1^{\mathrm{a}} \\
-0.5 \pm 0.1^{\mathrm{a}} \\
0.2 \pm 0.1^{\mathrm{a}}\end{array}$ & $\begin{array}{l}\text { not } \\
\text { reported }\end{array}$ & $\begin{array}{l}\text { not } \\
\text { reported }\end{array}$ & $\begin{array}{l}\text { not } \\
\text { reported }\end{array}$ & 2 \\
\hline $\begin{array}{l}\text { Dejager } \\
\text { et al. [43], } \\
2007\end{array}$ & $\begin{array}{l}\text { Vildagliptin } 50 \mathrm{mg} \text { once daily } \\
\text { Vildagliptin } 50 \mathrm{mg} \text { twice daily } \\
\text { Vildagliptin } 100 \mathrm{mg} \text { once daily } \\
\text { Placebo }\end{array}$ & $\begin{array}{l}8.2 \pm 0.1^{\mathrm{a}} \\
8.6 \pm 0.1^{\mathrm{a}} \\
8.4 \pm 0.1^{\mathrm{a}} \\
8.4 \pm 0.1^{\mathrm{a}}\end{array}$ & $\begin{array}{l}\text { not } \\
\text { reported }\end{array}$ & $\begin{array}{l}-0.8 \pm 0.1^{\mathrm{a}} \\
-0.8 \pm 0.1^{\mathrm{a}} \\
-0.9 \pm 0.1^{\mathrm{a}} \\
-0.3 \pm 0.1^{\mathrm{a}}\end{array}$ & $\begin{array}{r}104 \\
90 \\
92 \\
94\end{array}$ & $\begin{array}{l}\text { not } \\
\text { reported }\end{array}$ & $\begin{array}{r}1.8 \pm 0.4 \\
-0.3 \pm 0.4 \\
-0.8 \pm 0.4 \\
-1.4 \pm 0.4\end{array}$ & 3 \\
\hline $\begin{array}{l}\text { Garber } \\
\text { et al. [44], } \\
2007\end{array}$ & $\begin{array}{l}\text { Vildagliptin } 50 \mathrm{mg} \text { once daily } \\
\text { Vildagliptin } 100 \mathrm{mg} \text { once daily } \\
\text { Placebo }\end{array}$ & $\begin{array}{l}8.6 \pm 1.0 \\
8.7 \pm 1.2 \\
8.7 \pm 1.2\end{array}$ & $\begin{array}{l}7.7 \pm 0.1^{\mathrm{a}} \\
7.5 \pm 0.1^{\mathrm{a}} \\
8.1 \pm 0.1^{\mathrm{a}}\end{array}$ & & $\begin{array}{l}\text { not } \\
\text { reported }\end{array}$ & $\begin{array}{l}\text { not } \\
\text { reported }\end{array}$ & $\begin{array}{l}\text { not } \\
\text { reported }\end{array}$ & 1 \\
\hline $\begin{array}{l}\text { Garber } \\
\text { et al. [45], } \\
2008\end{array}$ & $\begin{array}{l}\text { Vildagliptin } 50 \text { mg once daily } \\
\text { Vildagliptin } 100 \mathrm{mg} \text { once daily } \\
\text { Placebo }\end{array}$ & $\begin{array}{l}8.5 \pm 0.9 \\
8.6 \pm 1.0 \\
8.5 \pm 1.0\end{array}$ & $\begin{array}{l}\text { not } \\
\text { reported }\end{array}$ & $\begin{array}{l}-0.58 \pm 0.10 \\
-0.63 \pm 0.09 \\
-0.07 \pm 0.09\end{array}$ & $\begin{array}{l}91.5 \pm 1.6 \\
87.3 \pm 1.6 \\
89.4 \pm 1.6\end{array}$ & $\begin{array}{l}\text { not } \\
\text { reported }\end{array}$ & $\begin{array}{r}1.3 \pm 0.3 \\
-0.1 \pm 0.3 \\
-0.4 \pm 0.3\end{array}$ & 8 \\
\hline $\begin{array}{l}\text { Goodman } \\
\text { et al. [46], } \\
2009\end{array}$ & $\begin{array}{l}\text { Vildagliptin } 100 \mathrm{mg} \text { once daily a.m. } \\
\text { Vildagliptin } 100 \mathrm{mg} \text { once daily p.m. } \\
\text { Vildagliptin total } \\
\text { Placebo once daily }\end{array}$ & $\begin{array}{l}8.5 \pm 1 \\
8.5 \pm 0.9 \\
8.5 \pm 1 \\
8.7 \pm 1.1\end{array}$ & $\begin{array}{l}\text { not } \\
\text { reported }\end{array}$ & $\begin{array}{l}-0.66 \pm 0.1^{\mathrm{a}} \\
-0.53 \pm 0.1^{\mathrm{a}} \\
\text { not reported } \\
0.17 \pm 0.1^{\mathrm{a}}\end{array}$ & $\begin{array}{l}\text { not } \\
\text { reported }\end{array}$ & $\begin{array}{l}\text { not } \\
\text { reported }\end{array}$ & $\begin{array}{l}\text { not } \\
\text { reported }\end{array}$ & 2 \\
\hline $\begin{array}{l}\text { Kikuchi } \\
\text { et al. [47], } \\
2009\end{array}$ & $\begin{array}{l}\text { Vildagliptin } 10 \mathrm{mg} \text { twice daily } \\
\text { Vildagliptin } 25 \mathrm{mg} \text { twice daily } \\
\text { Vildagliptin } 50 \mathrm{mg} \text { twice daily } \\
\text { Placebo twice daily }\end{array}$ & $\begin{array}{l}7.4 \pm 0.8 \\
7.4 \pm 0.9 \\
7.4 \pm 0.8 \\
7.4 \pm 0.8\end{array}$ & $\begin{array}{l}6.8 \pm 0.1^{\mathrm{a}} \\
6.7 \pm 0.1^{\mathrm{a}} \\
6.5 \pm 0.1^{\mathrm{a}} \\
7.6 \pm 0.1^{\mathrm{a}}\end{array}$ & $\begin{array}{r}-0.53 \\
-0.67 \\
-0.92 \\
0.28\end{array}$ & $\begin{array}{l}64.2 \pm 10.4 \\
63.6 \pm 9.7 \\
62.7 \pm 9.3 \\
63.8 \pm 10.1\end{array}$ & $\begin{array}{l}\text { not } \\
\text { reported }\end{array}$ & $\begin{array}{r}-0.2 \pm 1.5 \\
0.2 \pm 1.3 \\
0.5 \pm 1.4 \\
-0.5 \pm 1.1\end{array}$ & 5 \\
\hline $\begin{array}{l}\text { Pi-Sunyer } \\
\text { et al. [48], } \\
2007\end{array}$ & $\begin{array}{l}\text { Vildagliptin } 50 \mathrm{mg} \text { once daily } \\
\text { Vildagliptin } 50 \mathrm{mg} \text { twice daily } \\
\text { Vildagliptin } 100 \mathrm{mg} \text { once daily } \\
\text { Placebo }\end{array}$ & $\begin{array}{l}8.3 \pm 0.1^{\mathrm{a}} \\
8.4 \pm 0.1^{\mathrm{a}} \\
8.3 \pm 0.1^{\mathrm{a}} \\
8.5 \pm 0.1^{\mathrm{a}}\end{array}$ & $\begin{array}{l}7.9 \pm 0.2^{\mathrm{a}} \\
7.7 \pm 0.2^{\mathrm{a}} \\
7.5 \pm 0.1^{\mathrm{a}} \\
8.4 \pm 0.2^{\mathrm{a}}\end{array}$ & $\begin{array}{r}-0.5 \pm 0.1^{\mathrm{a}} \\
-0.7 \pm 0.1^{\mathrm{a}} \\
-0.8 \pm 0.1^{\mathrm{a}} \\
0.0 \pm 0.1^{\mathrm{a}}\end{array}$ & $\begin{array}{l}90.4 \pm 2.4^{\mathrm{a}} \\
90.4 \pm 2.1^{\mathrm{a}} \\
90.5 \pm 2.1^{\mathrm{a}} \\
92.6 \pm 2.5^{\mathrm{a}}\end{array}$ & $\begin{array}{l}90.1 \pm 2.4^{\mathrm{a}} \\
90.5 \pm 2.1^{\mathrm{a}} \\
90.2 \pm 2.2^{\mathrm{a}} \\
91.1 \pm 2.4^{\mathrm{a}}\end{array}$ & $\begin{array}{l}-0.4 \pm 0.4^{\mathrm{a}} \\
-0.0 \pm 0.4^{\mathrm{a}} \\
-0.4 \pm 0.3^{\mathrm{a}} \\
-1.4 \pm 0.4^{\mathrm{a}}\end{array}$ & 0 \\
\hline $\begin{array}{l}\text { Scherbaum } \\
\text { et al. [49], } \\
2008\end{array}$ & $\begin{array}{l}\text { Vildagliptin } 50 \mathrm{mg} \text { once daily } \\
\text { Placebo }\end{array}$ & $\begin{array}{l}6.7 \pm 0.4 \\
6.7 \pm 0.4\end{array}$ & $\begin{array}{l}\text { not } \\
\text { reported }\end{array}$ & $\begin{array}{r}-0.2 \pm 0.1 \\
0.1 \pm 0.1\end{array}$ & $\begin{array}{l}86 \pm 1.2 \\
85 \pm 1.4\end{array}$ & $\begin{array}{l}\text { not } \\
\text { reported }\end{array}$ & $\begin{array}{l}-0.5 \pm 0.3 \\
-0.2 \pm 0.3\end{array}$ & 0 \\
\hline $\begin{array}{l}\text { Mimori } \\
\text { et al. [50], } \\
2006\end{array}$ & $\begin{array}{l}\text { Vildagliptin } 10 \mathrm{mg} \text { twice daily } \\
\text { Vildagliptin } 25 \mathrm{mg} \text { twice daily } \\
\text { Vildagliptin } 50 \mathrm{mg} \text { twice daily } \\
\text { Placebo }\end{array}$ & $\begin{array}{l}7.4 \pm 0.8 \\
7.4 \pm 0.9 \\
7.4 \pm 0.8 \\
7.4 \pm 0.7\end{array}$ & $\begin{array}{l}\text { not } \\
\text { reported }\end{array}$ & $\begin{array}{l}0.53 \pm 0.07 \\
0.67 \pm 0.07 \\
0.92 \pm 0.07 \\
0.28 \pm 0.07\end{array}$ & $\begin{array}{l}\text { not } \\
\text { reported }\end{array}$ & $\begin{array}{l}\text { not } \\
\text { reported }\end{array}$ & $\begin{array}{l}\text { not } \\
\text { reported }\end{array}$ & 5 \\
\hline
\end{tabular}

${ }^{\text {a }}$ Means \pm SEM, all other results are means \pm SD. 
The impact of major adjustment variables on these pooled estimates was assessed using meta-regression [51]. These covariates included (1) study duration, (2) mean age, (3) gender, (4) duration of diabetes, (5) mean $\mathrm{HbA}_{1 \mathrm{c}}$ level at baseline, (6) mean weight at baseline and (7) whether the treatment was given as monotherapy or as add-on. However, these adjustments were only performed when data were available to run the model.

The metaregression model relates the treatment effect to the study level covariates, assuming a normal distribution for the residual errors. The estimated between-studies variance is reported for each model and is a measure of the residual heterogeneity once having adjusted for the covariates. An adjusted estimate of the difference between each treatment and placebo is reported for each analysis, alongside p values and 95\% two-sided CIs. All analyses were performed in Stata SE version 8.2.

\section{Results}

\section{Study/Patient Characteristics}

There were 8 trials on exenatide, 7 on liraglutide, 12 on sitagliptin and 11 on vildagliptin. Descriptions of the studies and of the patient characteristics are presented in the Appendix.

Of the 38 studies that were reviewed in depth, 2 had a duration longer than 30 weeks, both of which were vildagliptin trials. Trials on exenatide ranged in duration from 4 to 30 weeks, on liraglutide from 12 to 26 weeks, on sitagliptin from 12 to 30 weeks and on vildagliptin from 12 to 52 weeks. All study arms were directly compared with placebo; $34 / 38$ were double-blind while $4 / 38$ were tripleblind (all were exenatide trials).

In terms of patient characteristics, the mean age of patients across all trials ranged from 50.9 to 62.9 years. For exenatide patients, the mean age was $54.0 \pm 1.3$ years, for those on liraglutide $56.6 \pm 0.8$ years, sitagliptin $54.9 \pm$ 1.4 years and vildagliptin $56.4 \pm 3.3$ years. Gender distribution was also similar across trials. For example, the proportion of male participants in the exenatide trials ranged from 42 to $60 \%$, for liraglutide from 50 to $67 \%$, for sitagliptin from 45 to $62 \%$ and for vildagliptin from 47 to $67 \%$. There were also variations in the duration of the diabetes diagnosis: exenatide 1.7-8.9 years; liraglutide 4.5-9.0 years; sitagliptin $2.0-8.8$ years; vildagliptin $2.2-$ 7.2 years.

\section{Glycemic Control}

The results on glycemic control are presented in table 2. Both DPP-4 inhibitors, sitagliptin and vildagliptin, were shown to produce a statistically significant reduction in $\mathrm{HbA}_{1 \mathrm{c}}$ compared with placebo. The direct random-effect meta-analysis of randomized controlled trials
Table 2. Unadjusted results of $\mathrm{HbA}_{1 \mathrm{c}}$ comparing each study treatment with placebo

\begin{tabular}{lcccc}
\hline $\begin{array}{l}\text { Change from baseline } \\
\text { in } \mathrm{HbA}_{1 \mathrm{c}} \text { levels: } \\
\text { metaregression }\end{array}$ & $\begin{array}{l}\text { Number } \\
\text { of RCTs }\end{array}$ & WMD & $95 \%$ CI & $\begin{array}{l}\mathrm{p} \\
\text { value }\end{array}$ \\
\hline $\begin{array}{l}\text { Vildagliptin } \\
\text { Sitagliptin }\end{array}$ & 11 & -0.67 & -0.83 to -0.52 & $<0.001$ \\
$\begin{array}{l}\text { Exenatide } \\
\text { Liraglutide }\end{array}$ & 8 & -0.79 & -0.93 to -0.65 & $<0.001$ \\
& 7 & -0.75 & -0.83 to -0.67 & $<0.001$ \\
& & -1.03 & -1.16 to -0.90 & $<0.001$ \\
\hline
\end{tabular}

RCT $=$ Randomized controlled trial. comparing vildagliptin with placebo showed a statistically significant difference in $\mathrm{HbA}_{1 \mathrm{c}}$ decline from baseline favoring vildagliptin (unadjusted model: WMD = $-0.67,95 \% \mathrm{CI}=-0.83$ to $-0.52, \mathrm{p}<0.001)$. Controlling for the covariates did not impact on the results.

Similar $\mathrm{HbA}_{1 \mathrm{c}}$ efficacy results were found when sitagliptin was compared with placebo (unadjusted model: $\mathrm{WMD}=-0.79,95 \% \mathrm{CI}=-0.93$ to $-0.65, \mathrm{p}<0.001$ ), with a significantly high level of heterogeneity detected in the analysis $\left(\chi^{2} \mathrm{p}<0.001\right)$. In the adjusted analyses, 2 covariates were found to be statistically significant in the models: baseline weight (WMD $=-0.74,95 \% \mathrm{CI}=-0.83$ to $-0.65, \mathrm{p}<0.001)$ and baseline $\mathrm{HbA}_{1 \mathrm{c}}$ level $(\mathrm{WMD}=-0.80$, $95 \% \mathrm{CI}=-0.92$ to $-0.68, \mathrm{p}<0.001)$.

When exenatide and liraglutide were compared against placebo, both treatments showed a statistically significant difference in $\mathrm{HbA}_{1 c}$ decrease from baseline. Two separate direct fixed-effect meta-analyses showed statistically significant differences in $\mathrm{HbA}_{1 c}$ decrease from baseline favoring exenatide (unadjusted model: $\mathrm{WMD}=-0.75,95 \% \mathrm{CI}=-0.83$ to $-0.67, \mathrm{p}<0.001)$, and favoring liraglutide (unadjusted model: $\mathrm{WMD}=-1.03$, $95 \% \mathrm{CI}=-1.16$ to $-0.90, \mathrm{p}<0.001$ ), compared with placebo. In the adjusted analyses for exenatide, controlling for whether exenatide was given as monotherapy or in combination with another treatment provided the most variability, but even this estimate fell within the boundaries of the unadjusted model CI (WMD $=-0.84,95 \%$ $\mathrm{CI}=-0.95$ to $-0.73, \mathrm{p}<0.001)$. Two other covariates were found to be statistically significant in the exenatide model: age (WMD $=-0.74,95 \% \mathrm{CI}=-0.82$ to $-0.67, \mathrm{p}<0.001)$ and duration of diabetes $(\mathrm{WMD}=-0.74,95 \% \mathrm{CI}=-0.82$ to $-0.66, \mathrm{p}<0.001)$. In the adjusted analyses for liraglutide, no covariates were found to be statistically significant. 
Table 3. Unadjusted results of weight comparing each study treatment with placebo

\begin{tabular}{lccc}
\hline Metaregression & WMD & $95 \%$ CI & p value \\
\hline Vildagliptin & 0.56 & 0.27 to 0.84 & $<0.001$ \\
Sitagliptin & 0.60 & 0.33 to 0.87 & $<0.001$ \\
Exenatide & -1.10 & -1.32 to -0.88 & $<0.001$ \\
Liraglutide & -0.82 & -1.92 to 0.27 & 0.142 \\
\hline
\end{tabular}

\section{Weight}

The results for weight are presented in table 3. A direct meta-analysis of studies comparing sitagliptin versus placebo and reporting data on changes in weight indicated that there was a statistically significant weight gain with sitagliptin compared with placebo (unadjusted model: $\mathrm{WMD}=0.60,95 \% \mathrm{CI}=0.33-0.87, \mathrm{p}<0.001)$. When we controlled for the covariates in the adjusted model, study duration was the only variable that was statistically significant, with a positive correlation between treatments $(\mathrm{WMD}=0.67,95 \% \mathrm{CI}=0.48-0.87, \mathrm{p}<0.001)$.

The analyses indicated a statistically significant weight gain with vildagliptin (unadjusted model: $\mathrm{WMD}=0.56$, 95\% CI $=0.27-0.84, \mathrm{p}<0.001$ ). Exenatide (unadjusted model: $\mathrm{WMD}=-1.10,95 \% \mathrm{CI}=-1.32$ to $-0.88, \mathrm{p}<0.001)$ and liraglutide (unadjusted model: WMD $=-0.82,95 \%$ $\mathrm{CI}=-1.92$ to $-0.27, \mathrm{p}=0.142$; table 3 ) both exhibited reduction in weight. The most remarkable result is the average weight reduction of $1.10 \mathrm{~kg}$ observed with exenatide. In the adjusted models for liraglutide, exenatide and vildagliptin, none of the covariates were statistically significant.

\section{Hypoglycemia}

The meta-analysis of vildagliptin versus placebo showed a nonstatistically significant increase in the number of patients who reported hypoglycemic episodes in comparison to those who received a placebo [relative risk $(R R)=1.52,95 \% \mathrm{CI}=0.80-2.88, \mathrm{p}=0.196]$. As for sitagliptin, patients under this treatment were $156 \%$ more likely to experience some hypoglycemia in comparison to placebo patients (unadjusted model: $\mathrm{RR}=2.56,95 \% \mathrm{CI}=$ $1.23-5.33, \mathrm{p}=0.01)$. When adjusted for covariates, age was the only variable found to be statistically significant $(\mathrm{RR}=1.84,95 \% \mathrm{CI}=1.02-3.34, \mathrm{p}=0.044)$.

For the incretin mimetics, the analysis showed that patients treated with exenatide were $140 \%$ more likely to experience some hypoglycemia than patients treated with placebo, and this difference was statistically signifi- cant $(\mathrm{RR}=2.40,95 \% \mathrm{CI}=1.39-4.11, \mathrm{p}=0.002)$. Patients treated with liraglutide were $69 \%$ more likely to experience some hypoglycemia than patients treated with placebo. This difference just reached significance at the $95 \%$ probability level $(\mathrm{RR}=1.69,95 \% \mathrm{CI}=1.00-2.86, \mathrm{p}=$ $0.050)$. No covariates were statistically significant in the adjusted models.

\section{Discussion}

This meta-analysis assessed the clinical efficacy and safety of incretin mimetics and DDP-4 inhibitors in T2DM patients compared with placebo. In the analysis, we ran a direct meta-analysis of each treatment versus placebo, and assessed the impact of adjustment variables on the results of the meta-analyses. When there was a significant moderate to high level of heterogeneity in this meta-analysis, the random-effect model was used to pool the data, and analyses were conducted controlling for heterogeneity by adjusting for key study covariates (age, study duration, duration of diabetes, gender and treatment as monotherapy or in combination). There were only very few instances where the covariates had a statistically significant impact on the outcomes. The present analysis therefore represents the best synthesis of the current published evidence on the clinical efficacy and safety of each of the treatments in comparison to placebo.

The analysis has indicated that treatment with either incretin mimetics (exenatide, liraglutide) or DPP-4 inhibitors (vildagliptin, sitagliptin) leads to reasonable glycemic control in comparison to placebo. However, there were some differences in changes in patient weight and the risk of experiencing hypoglycemic episodes. In terms of clinical efficacy, as measured by the level of reduction in $\mathrm{HbA}_{1 \mathrm{c}}$, and in comparison to placebo, liraglutide ranked highest (a reduction of 1\% across trials of 12-26 weeks), followed by sitagliptin (a reduction of $0.79 \%$ across trials of $20-30$ weeks), exenatide (a reduction of $0.75 \%$ across trials of 4-30 weeks) and vildagliptin (a reduction of $0.67 \%$ across trials of 12-52 weeks). The findings from our analyses contrast with those of Chia and Egan [52], who reported that liraglutide lowered $\mathrm{HbA}_{1 \mathrm{c}}$ by $1.5 \%$ in a 14-week study (with no evidence from phase III trials), sitagliptin monotherapy lowered $\mathrm{HbA}_{1 \mathrm{c}}$ by $0.6-0.7 \%$ after 54 weeks, and vildagliptin by $0.9-1.4 \%$ after 24 weeks. A possible explanation for this difference could be that more trials were included in our review and that in our analyses a pooled estimate of the effect of each treatment versus placebo for $\mathrm{HbA}_{1 \mathrm{c}}$ was obtained from all selected trials on each treatment. 
As far as safety outcomes are concerned, our analysis indicated a statistically significant reduction in weight with exenatide and liraglutide as compared with placebo. A statistically significant weight gain was observed with use of sitagliptin and vildagliptin. The analyses also showed that patients on sitagliptin and exenatide were statistically significantly more likely to experience hypoglycemic episodes compared with patients on placebo. One should note the importance of achieving glycemic control while reducing adverse events such as hypoglycemia or weight gain. Evidence in the literature points to fear of hypoglycemia as a major barrier to implementation of intensive therapy and has adversely affected adherence to the prescribed medication regimen [53]. In addition, the specter of weight gain can increase diabetic morbidity and mortality when acting as a psychological barrier to the initiation or intensification of treatment, or affecting adherence to prescribed regimens [54].

A major limitation of our analysis was that the trials included exhibited a wide variation in study duration. Trials on exenatide ranged from 4 to 30 weeks, those on liraglutide from 12 to 26 weeks, on sitagliptin from 12 to 30 weeks and on vildagliptin from 12 to 52 weeks. To con- trol for this, we ran adjusted models with study duration as a covariate for the $\mathrm{HbA}_{1 \mathrm{c}}$, weight and hypoglycemia outcomes. Study duration was found to have a statistically significant impact only on weight gain for patients on sitagliptin $(\mathrm{WMD}=0.67,95 \% \mathrm{CI}=0.48-0.87, \mathrm{p}<$ 0.001). In addition, longer study duration does not seem to be associated with a cumulatively greater number of patient-reported hypoglycemic episodes for any of the treatments under investigation and appears to have no statistically significant impact on reporting hypoglycemic episodes. The data also suggest that the relationship between study duration and the number of hypoglycemic episodes may be nonlinear. For example, while Ristic et al. [41] reported the total number of hypoglycemic episodes reported by vildagliptin patients to be 14 in a 12week trial, Garber et al. [44] reported it to be 1 in a 24week trial. Raz et al. [36] reported the total number of hypoglycemic episodes in patients on sitagliptin to be 5 in a 30-week trial, while Goldstein et al. [31] reported 7 in a 24-week trial. One may therefore presume that the difference in study duration does not account for the wide variation in hypoglycemia event rates and/or variations in reduction of $\mathrm{HbA}_{1 \mathrm{c}}$ and weight changes.

\section{Appendix 1}

Study Description and Patient Characteristics

Vildagliptin

\begin{tabular}{|c|c|c|c|c|}
\hline $\begin{array}{l}\text { Study } \\
\text { reference }\end{array}$ & Aim & $\begin{array}{l}\text { Study } \\
\text { design }\end{array}$ & Participants & Intervention \\
\hline $\begin{array}{l}\text { Ahren } \\
\text { et al. [40], } \\
2004\end{array}$ & $\begin{array}{l}\text { To assess } 12 \text { - and } 52 \text {-week efficacy of vildagliptin } \\
\text { versus placebo in patients with T2DM } \\
\text { continuing metformin treatment }\end{array}$ & $\begin{array}{l}\text { Double- } \\
\text { blind RCT }\end{array}$ & $\begin{array}{l}107 \text { patients, mean age } 56.9 \text { years, } \\
62 \% \text { male, mean duration of diabetes } \\
5.6 \text { years; study duration } 12 \text { weeks }\end{array}$ & Vildagliptin 50 mg q.d. \\
\hline $\begin{array}{l}\text { Ristic } \\
\text { et al. [41], } \\
2005\end{array}$ & $\begin{array}{l}\text { To establish a dose of vildagliptin that was } \\
\text { effective in reducing } \mathrm{HbA}_{1 \mathrm{c}} \text { levels and was well } \\
\text { tolerated in patients with } \mathrm{T} 2 \mathrm{DM}\end{array}$ & $\begin{array}{l}\text { Double- } \\
\text { blind RCT }\end{array}$ & $\begin{array}{l}279 \text { patients, mean age } 56.1 \text { years, } \\
54 \% \text { male, mean duration of diabetes } \\
2.9 \text { years; study duration } 12 \text { weeks }\end{array}$ & $\begin{array}{l}\text { Vildagliptin } 25 \text { mg b.i.d., } \\
25 \text { mg q.d., } 50 \text { mg q.d. or } \\
100 \text { mg q.d. }\end{array}$ \\
\hline $\begin{array}{l}\text { Bosi } \\
\text { et al. [42], } \\
2007\end{array}$ & $\begin{array}{l}\text { To evaluate the efficacy and safety of vildagliptin } \\
\text { added to metformin }\end{array}$ & $\begin{array}{l}\text { Double- } \\
\text { blind RCT }\end{array}$ & $\begin{array}{l}544 \text { patients, mean age } 54.2 \text { years, } \\
57 \% \text { male, mean duration of diabetes } \\
6.3 \text { years; study duration } 24 \text { weeks }\end{array}$ & $\begin{array}{l}\text { Vildagliptin } 50 \text { mg q.d. or } \\
100 \text { mg q.d. }\end{array}$ \\
\hline $\begin{array}{l}\text { Dejager } \\
\text { et al. [43], } \\
2007\end{array}$ & $\begin{array}{l}\text { To assess the efficacy and tolerability of } \\
\text { vildagliptin }\end{array}$ & $\begin{array}{l}\text { Double- } \\
\text { blind RCT }\end{array}$ & $\begin{array}{l}632 \text { patients, mean age } 53.5 \text { years, } \\
47 \% \text { male, mean duration of diabetes } \\
2.90 \text { years; study duration } 24 \text { weeks }\end{array}$ & $\begin{array}{l}\text { Vildagliptin } 50 \text { mg q.d., } \\
50 \text { mg b.i.d. or } 100 \text { mg q.d. }\end{array}$ \\
\hline $\begin{array}{l}\text { Garber } \\
\text { et al. [44], } \\
2007\end{array}$ & $\begin{array}{l}\text { To assess the efficacy and tolerability of } \\
\text { vildagliptin in combination with pioglitazone in } \\
\text { patients with T2DM }\end{array}$ & $\begin{array}{l}\text { Double- } \\
\text { blind RCT }\end{array}$ & $\begin{array}{l}463 \text { patients, mean age } 54.3 \text { years, } \\
50 \% \text { male, mean duration of diabetes } \\
4.7 \text { years; study duration } 24 \text { weeks }\end{array}$ & Vildagliptin 50 or 100 mg q.d. \\
\hline $\begin{array}{l}\text { Garber } \\
\text { et al. [45], } \\
2008\end{array}$ & $\begin{array}{l}\text { To compare the efficacy and tolerability of } \\
\text { vildagliptin versus placebo in patients with } \\
\text { T2DM who are inadequately controlled with } \\
\text { prior sulfonylurea monotherapy }\end{array}$ & $\begin{array}{l}\text { Double- } \\
\text { blind RCT }\end{array}$ & $\begin{array}{l}515 \text { patients, mean age } 58.2 \text { years, } \\
59 \% \text { male, mean duration of diabetes } \\
7.2 \text { years; study duration } 24 \text { weeks }\end{array}$ & $\begin{array}{l}\text { Vildagliptin } 50 \mathrm{mg} \text { q.d. or } \\
\text { b.i.d. }\end{array}$ \\
\hline
\end{tabular}




\begin{tabular}{|c|c|c|c|c|}
\hline $\begin{array}{l}\text { Goodman } \\
\text { et al. [46], } \\
2009\end{array}$ & $\begin{array}{l}\text { To demonstrate that } \mathrm{HbA}_{1 \mathrm{c}} \text { reduction with } \\
\text { vildagliptin } 100 \mathrm{mg} \text { q.d. a.m. dosing is superior } \\
\text { to placebo }\end{array}$ & $\begin{array}{l}\text { Double- } \\
\text { blind RCT }\end{array}$ & $\begin{array}{l}370 \text { patients, mean age } 54.8 \text { years, } \\
58 \% \text { male; study duration } 24 \text { weeks }\end{array}$ & $\begin{array}{l}\text { Vildagliptin } 100 \text { mg a.m. and } \\
100 \text { mg p.m. }\end{array}$ \\
\hline $\begin{array}{l}\text { Kikuchi } \\
\text { et al. [47], } \\
2009\end{array}$ & $\begin{array}{l}\text { To assess the efficacy and tolerability of } \\
\text { vildagliptin (10, } 25 \text { or } 50 \mathrm{mg} \text { b.i.d.) in Japanese } \\
\text { patients with T2DM }\end{array}$ & $\begin{array}{l}\text { Double- } \\
\text { blind RCT }\end{array}$ & $\begin{array}{l}291 \text { patients, mean age } 59.0 \text { years, } \\
67 \% \text { male, mean duration of diabetes } \\
5.2 \text { years; study duration } 12 \text { weeks }\end{array}$ & $\begin{array}{l}\text { Vildagliptin 10, } 25 \text { or } 50 \mathrm{mg} \\
\text { b.i.d. }\end{array}$ \\
\hline $\begin{array}{l}\text { Pi-Sunyer } \\
\text { et al. [48], } \\
2007\end{array}$ & $\begin{array}{l}\text { To assess the efficacy and tolerability of } \\
\text { vildagliptin }\end{array}$ & $\begin{array}{l}\text { Double- } \\
\text { blind RCT }\end{array}$ & $\begin{array}{l}354 \text { patients, mean age } 51.2 \text { years, } \\
55 \% \text { male, mean duration of diabetes } \\
2.2 \text { years; study duration } 24 \text { weeks }\end{array}$ & $\begin{array}{l}\text { Vildagliptin } 50 \text { mg q.d., } \\
50 \text { mg b.i.d. or } 100 \text { mg q.d. }\end{array}$ \\
\hline $\begin{array}{l}\text { Scherbaum } \\
\text { et al. [49], } \\
2008\end{array}$ & $\begin{array}{l}\text { To assess the efficacy and tolerability of } \\
\text { vildagliptin in drug-naive patients with T2DM } \\
\text { and mild hyperglycemia }\end{array}$ & $\begin{array}{l}\text { Double- } \\
\text { blind RCT }\end{array}$ & $\begin{array}{l}306 \text { patients, mean age } 62.9 \text { years, } \\
57 \% \text { male, mean duration of diabetes } \\
2.6 \text { years; study duration } 52 \text { weeks }\end{array}$ & Vildagliptin 50 mg q.d. \\
\hline $\begin{array}{l}\text { Mimori } \\
\text { et al. [50], } \\
2006\end{array}$ & $\begin{array}{l}\text { To assess the efficacy and tolerability of } \\
\text { vildagliptin }\end{array}$ & $\begin{array}{l}\text { Double- } \\
\text { blind RCT }\end{array}$ & 219 patients, mean age 59 years & $\begin{array}{l}\text { Vildagliptin } 10 \mathrm{mg} \text { twice } \\
\text { daily, } 25 \mathrm{mg} \text { twice daily or } \\
50 \mathrm{mg} \text { twice daily, placebo }\end{array}$ \\
\hline
\end{tabular}

\section{Sitagliptin}

\begin{tabular}{|c|c|c|c|}
\hline $\begin{array}{l}\text { Study } \\
\text { reference }\end{array}$ & Aim & Study design & Participants \\
\hline $\begin{array}{l}\text { Scott } \\
\text { et al. }[28] \\
2008\end{array}$ & $\begin{array}{l}\text { To assess the addition of sitagliptin to ongoing metformin } \\
\text { therapy in patients with T2DM who were inadequately } \\
\text { controlled with } \mathrm{HbA}_{1 \mathrm{c}} 7-11 \% \text { on metformin monotherapy }\end{array}$ & $\begin{array}{l}\text { Double-blind } \\
\text { RCT }\end{array}$ & $\begin{array}{l}273 \text { patients, mean age } 55.2 \text { years, } 57 \% \text { male, mean } \\
\text { duration of diabetes } 5.2 \text { years; study duration } 18 \text { weeks }\end{array}$ \\
\hline $\begin{array}{l}\text { Aschner et al. } \\
{[29], 2006}\end{array}$ & $\begin{array}{l}\text { To examine the efficacy and safety of sitagliptin as } \\
\text { monotherapy in patients with T2DM }\end{array}$ & $\begin{array}{l}\text { Double-blind } \\
\text { RCT }\end{array}$ & $\begin{array}{l}741 \text { patients, mean age } 54.2 \text { years, } 51.7 \% \text { male, mean } \\
\text { duration of diabetes } 4.4 \text { years; study duration } 24 \text { weeks }\end{array}$ \\
\hline $\begin{array}{l}\text { Charbonnel } \\
\text { et al. [30], } \\
2006\end{array}$ & $\begin{array}{l}\text { To assess the efficacy and safety of sitagliptin, added to } \\
\text { ongoing metformin therapy, in patients with T2DM who } \\
\text { had inadequate glycemic control with metformin alone }\end{array}$ & $\begin{array}{l}\text { Double-blind } \\
\text { RCT }\end{array}$ & $\begin{array}{l}701 \text { patients, mean age } 54.5 \text { years, } 57.1 \% \text { male, mean } \\
\text { duration of diabetes } 6.2 \text { years; study duration } 24 \text { weeks }\end{array}$ \\
\hline $\begin{array}{l}\text { Goldstein } \\
\text { et al. [31], } \\
2007\end{array}$ & $\begin{array}{l}\text { To assess the efficacy and safety of initial combination } \\
\text { therapy with sitagliptin and metformin in patients with } \\
\text { T2DM and inadequate glycemic control by diet and exercise }\end{array}$ & $\begin{array}{l}\text { Double-blind } \\
\text { RCT }\end{array}$ & $\begin{array}{l}\text { 1,091 patients, mean age } 53.6 \text { years, } 51 \% \text { male; study } \\
\text { duration } 24 \text { weeks }\end{array}$ \\
\hline $\begin{array}{l}\text { Hanefeld et al. } \\
{[32], 2007}\end{array}$ & $\begin{array}{l}\text { To evaluate sitagliptin once daily monotherapy in a dose- } \\
\text { ranging study }\end{array}$ & $\begin{array}{l}\text { Double-blind } \\
\text { RCT }\end{array}$ & $\begin{array}{l}555 \text { patients, mean age } 55.1 \text { years, } 54.3 \% \text { male, mean } \\
\text { duration of diabetes } 4.5 \text { years; study duration } 18 \text { weeks }\end{array}$ \\
\hline $\begin{array}{l}\text { Hermansen } \\
\text { et al. [33], } \\
2007\end{array}$ & $\begin{array}{l}\text { To assess the efficacy and safety of sitagliptin in patients } \\
\text { with T2DM who had inadequate glycemic control while } \\
\text { on glimepiride alone or in combination with metformin }\end{array}$ & $\begin{array}{l}\text { Double-blind } \\
\text { RCT }\end{array}$ & $\begin{array}{l}441 \text { patients, mean age } 56.0 \text { years, } 53.1 \% \text { male, mean } \\
\text { duration of diabetes } 8.8 \text { years; study duration } 24 \text { weeks }\end{array}$ \\
\hline $\begin{array}{l}\text { Mohan } \\
\text { et al. [34], } \\
2009\end{array}$ & $\begin{array}{l}\text { To evaluate the efficacy and safety of sitagliptin as } \\
\text { monotherapy in Chinese, Indian and Korean patients with } \\
\text { T2DM inadequately controlled by diet and exercise }\end{array}$ & $\begin{array}{l}\text { Double-blind } \\
\text { RCT }\end{array}$ & $\begin{array}{l}530 \text { patients, mean age } 50.9 \text { years, } 57.7 \% \text { male, mean } \\
\text { duration of diabetes } 2.0 \text { years; study duration } 18 \text { weeks }\end{array}$ \\
\hline $\begin{array}{l}\text { Nonaka et al. } \\
{[35], 2008}\end{array}$ & $\begin{array}{l}\text { Efficacy and tolerability of sitagliptin was assessed in } \\
\text { Japanese patients with T2DM }\end{array}$ & $\begin{array}{l}\text { Double-blind } \\
\text { RCT }\end{array}$ & $\begin{array}{l}151 \text { patients, mean age } 55.3 \text { years, } 62.5 \% \text { male, mean } \\
\text { duration of diabetes } 4.1 \text { years; study duration } 12 \text { weeks }\end{array}$ \\
\hline $\begin{array}{l}\text { Raz } \\
\text { et al. [36], } \\
2008\end{array}$ & $\begin{array}{l}\text { To evaluate the efficacy and safety of sitagliptin as an add-on } \\
\text { to metformin therapy in patients with moderately severe } \\
\text { T2DM }\end{array}$ & $\begin{array}{l}\text { Double-blind } \\
\text { RCT }\end{array}$ & $\begin{array}{l}190 \text { patients, mean age } 54.8 \text { years, } 45.3 \% \text { male, mean } \\
\text { duration of diabetes } 7.9 \text { years; study duration } 30 \text { weeks }\end{array}$ \\
\hline $\begin{array}{l}\text { Scott } \\
\text { et al. [37], } \\
2007\end{array}$ & $\begin{array}{l}\text { To assess the efficacy and tolerability of sitagliptin, in } \\
\text { patients with T2DM who had inadequate glycemic control } \\
\text { by diet and exercise }\end{array}$ & $\begin{array}{l}\text { Double-blind } \\
\text { RCT }\end{array}$ & $\begin{array}{l}743 \text { patients, mean age } 55.5 \text { years, } 54 \% \text { male, mean } \\
\text { duration of diabetes } 4.6 \text { years; study duration } 12 \text { weeks }\end{array}$ \\
\hline $\begin{array}{l}\text { Rosenstock } \\
\text { et al. [38], } \\
2006\end{array}$ & $\begin{array}{l}\text { To assess the efficacy and tolerability of sitagliptin added to } \\
\text { ongoing pioglitazone therapy in patients with T2DM and } \\
\text { inadequate glycemic control }\end{array}$ & $\begin{array}{l}\text { Double-blind } \\
\text { RCT }\end{array}$ & $\begin{array}{l}535 \text { patients. mean age } 56.3 \text { years, } 55.5 \% \text { male, mean } \\
\text { duration of diabetes } 6.1 \text { years; study duration } 24 \text { weeks }\end{array}$ \\
\hline
\end{tabular}




\section{Exenatide}

\begin{tabular}{|c|c|c|c|c|c|}
\hline $\begin{array}{l}\text { Study } \\
\text { reference }\end{array}$ & Aim & $\begin{array}{l}\text { Study } \\
\text { design }\end{array}$ & Participants & Intervention & Treatment add-on \\
\hline $\begin{array}{l}\text { Zinman } \\
\text { et al. [13], } \\
2007\end{array}$ & $\begin{array}{l}\text { To compare the effects of exenatide } \\
\text { versus placebo on glycemic control }\end{array}$ & $\begin{array}{l}\text { Double- } \\
\text { blind RCT }\end{array}$ & $\begin{array}{l}233 \text { patients, mean age } \\
56 \text { years, } 55 \% \text { male, } \\
\text { mean duration of } \\
\text { diabetes } 7.7 \text { years; study } \\
\text { duration } 16 \text { weeks }\end{array}$ & $\begin{array}{l}\text { Subcutaneous abdominal injections of } \\
10 \mu \mathrm{g} \text { of exenatide b.i.d. }\end{array}$ & $\begin{array}{l}\text { Thiazolidinediones } \\
\text { (with or without } \\
\text { metformin) }\end{array}$ \\
\hline $\begin{array}{l}\text { De Fronzo } \\
\text { et al. [14], } \\
2005\end{array}$ & $\begin{array}{l}\text { To evaluate the ability of exenatide } \\
\text { to improve glycemic control in } \\
\text { patients with T2DM failing to } \\
\text { achieve glycemic control with } \\
\text { maximally effective metformin } \\
\text { doses }\end{array}$ & $\begin{array}{l}\text { Triple- } \\
\text { blind RCT }\end{array}$ & $\begin{array}{l}336 \text { patients, mean age } \\
53 \text { years, } 57 \% \text { male, } \\
\text { mean duration of } \\
\text { diabetes } 5.9 \text { years; study } \\
\text { duration } 30 \text { weeks }\end{array}$ & $\begin{array}{l}5 \mu g \text { s.c. exenatide b.i.d. for } 4 \text { weeks } \\
\text { followed by } 5 \mu \text { g or } 10 \mu g \text { s.c. } \\
\text { exenatide b.i.d. for } 26 \text { weeks }\end{array}$ & Metformin \\
\hline $\begin{array}{l}\text { Gao et al. } \\
{[15]} \\
2009\end{array}$ & $\begin{array}{l}\text { To evaluate the efficacy of } \\
\text { exenatide in Asian patients with } \\
\text { T2DM inadequately controlled } \\
\text { with oral agents }\end{array}$ & $\begin{array}{l}\text { Double- } \\
\text { blind RCT }\end{array}$ & $\begin{array}{l}465 \text { patients, mean age } \\
55 \text { years, } 44 \% \text { male, } \\
\text { mean duration of } \\
\text { diabetes } 8 \text { years; study } \\
\text { duration } 16 \text { weeks }\end{array}$ & $\begin{array}{l}5 \mu \mathrm{g} \text {, then } 10 \mu \mathrm{g} \text { b.i.d. for } 4 \text { and } 12 \\
\text { weeks }\end{array}$ & $\begin{array}{l}\text { Metformin alone or } \\
\text { with a sulfonylurea }\end{array}$ \\
\hline $\begin{array}{l}\text { Kendall } \\
\text { et al. [16], } \\
2005\end{array}$ & $\begin{array}{l}\text { To evaluate the effects of exenatide } \\
\text { in hyperglycemic patients with } \\
\text { T2DM unable to achieve glycemic } \\
\text { control with metformin/sulfonyl- } \\
\text { urea combination therapy }\end{array}$ & $\begin{array}{l}\text { Double- } \\
\text { blind RCT }\end{array}$ & $\begin{array}{l}733 \text { patients, mean age } \\
55 \text { years, } 52 \% \text { male, } \\
\text { mean duration of } \\
\text { diabetes } 8.9 \text { years; study } \\
\text { duration } 30 \text { weeks }\end{array}$ & $\begin{array}{l}5 \mu \text { g s.c. exenatide b.i.d. (arms A and } \\
\text { B) for } 4 \text { weeks; thereafter, arm A } \\
\text { remained at } 5 \mu \mathrm{g} \text { b.i.d. and arm B } \\
\text { escalated to } 10 \mu \mathrm{g} \text { b.i.d. }\end{array}$ & Metformin \\
\hline $\begin{array}{l}\text { Moretto } \\
\text { et al. [17], } \\
2008\end{array}$ & $\begin{array}{l}\text { To evaluate the efficacy and toler- } \\
\text { ability of exenatide monotherapy in } \\
\text { patients with T2DM naive to anti- } \\
\text { diabetic agents and whose disease } \\
\text { was inadequately controlled with } \\
\text { diet and exercise alone }\end{array}$ & $\begin{array}{l}\text { Double- } \\
\text { blind RCT }\end{array}$ & $\begin{array}{l}232 \text { patients, mean age } \\
54 \text { years, } 56 \% \text { male, } \\
\text { mean duration of } \\
\text { diabetes } 1.7 \text { years; study } \\
\text { duration } 24 \text { weeks }\end{array}$ & 5 or $10 \mu$ g s.c. exenatide b.i.d. & None \\
\hline $\begin{array}{l}\text { Poon } \\
\text { et al. [18], } \\
2005\end{array}$ & $\begin{array}{l}\text { To assess the dose dependencies of } \\
\text { the glucoregulatory effects and tol- } \\
\text { erability of exenatide when added } \\
\text { to diet and exercise or metformin } \\
\text { monotherapy in patients with } \\
\text { T2DM }\end{array}$ & $\begin{array}{l}\text { Triple- } \\
\text { blind RCT }\end{array}$ & $\begin{array}{l}156 \text { patients, mean age } \\
53 \text { years, } 43 \% \text { male, } \\
\text { mean duration of } \\
\text { diabetes } 3.9 \text { years; study } \\
\text { duration } 4 \text { weeks }\end{array}$ & $2.5,5.0,7.5$ or $10 \mu \mathrm{g}$ b.i.d. & Metformin \\
\hline $\begin{array}{l}\text { Buse } \\
\text { et al. [19], } \\
2004\end{array}$ & $\begin{array}{l}\text { To evaluate the ability of exenatide } \\
\text { to improve glycemic control in } \\
\text { patients with T2DM failing } \\
\text { maximally effective doses of a } \\
\text { sulfonylurea as monotherapy }\end{array}$ & $\begin{array}{l}\text { Triple- } \\
\text { blind RCT }\end{array}$ & $\begin{array}{l}377 \text { patients, mean age } \\
55 \text { years, } 60 \% \text { male, } \\
\text { mean duration of } \\
\text { diabetes } 6.2 \text { years; study } \\
\text { duration } 30 \text { weeks }\end{array}$ & $\begin{array}{l}4 \text { weeks at } 5 \mu \text { g s.c. exenatide b.i.d. } \\
\text { (arms A and B) or placebo; } \\
\text { subsequently, subjects in arm B were } \\
\text { escalated to } 10 \mu \text { g b.i.d. exenatide }\end{array}$ & $\begin{array}{l}\text { Sulfonylurea } \\
\text { therapy }\end{array}$ \\
\hline $\begin{array}{l}\text { Fineman } \\
\text { et al. [20], } \\
2003\end{array}$ & $\begin{array}{l}\text { To examine the activity and safety } \\
\text { of subcutaneously injected AC2993 } \\
\text { (exenatide) in patients withT2DM } \\
\text { currently treated with diet and/or } \\
\text { oral antidiabetic agents }\end{array}$ & $\begin{array}{l}\text { Triple- } \\
\text { blind RCT }\end{array}$ & $\begin{array}{l}109 \text { patients, mean age } \\
52 \text { years, } 54 \% \text { male; } \\
\text { study duration } 4 \text { weeks }\end{array}$ & $\begin{array}{l}\text { Exenatide } 0.08 \mu \mathrm{g} / \mathrm{kg} \text { s.c.; three } \\
\text { treatment groups: b.i.d. (breakfast and } \\
\text { dinner); b.i.d. (breakfast and bedtime); } \\
\text { t.i.d. (breakfast, dinner and bedtime) }\end{array}$ & $\begin{array}{l}\text { Regimen of } \\
\text { sulfonylurea or } \\
\text { metformin (alone or } \\
\text { in combination) }\end{array}$ \\
\hline
\end{tabular}




\section{Liraglutide}

\begin{tabular}{|c|c|c|c|c|}
\hline Study reference & Aim & Study design & Participants & Intervention \\
\hline $\begin{array}{l}\text { Zinman } \\
\text { et al. }[21] \\
2009\end{array}$ & $\begin{array}{l}\text { To determine the efficacy and safety of } \\
\text { liraglutide when added to metformin and } \\
\text { rosiglitazone in T2DM }\end{array}$ & $\begin{array}{l}\text { Double-blind } \\
\text { RCT }\end{array}$ & $\begin{array}{l}533 \text { patients, mean age } 55.1 \text { years, } \\
66 \% \text { male, mean duration of diabetes } \\
9 \text { years; study duration } 26 \text { weeks }\end{array}$ & Liraglutide 1.2 or $1.8 \mathrm{mg}$ \\
\hline $\begin{array}{l}\text { Madsbad } \\
\text { et al. [22], } \\
2004\end{array}$ & $\begin{array}{l}\text { To assess the efficacy and safety of liraglu- } \\
\text { tide after } 12 \text { weeks of treatment in T2DM } \\
\text { patients }\end{array}$ & $\begin{array}{l}\text { Double-blind } \\
\text { RCT }\end{array}$ & $\begin{array}{l}193 \text { patients, mean age } 56.7 \text { years, } \\
67 \% \text { male, mean duration of diabetes } \\
4.5 \text { years; study duration } 12 \text { weeks }\end{array}$ & $\begin{array}{l}\text { Liraglutide } 0.045,0.225,0.45 \text {, } \\
0.60 \text { or } 0.75 \mathrm{mg} \text { or open-label } \\
\text { glimepiride } 1-4 \mathrm{mg}\end{array}$ \\
\hline $\begin{array}{l}\text { Marre } \\
\text { et al. [23], } \\
2009\end{array}$ & $\begin{array}{l}\text { To compare the effects of combining } \\
\text { liraglutide }(0.6,1.2 \text { or } 1.8 \mathrm{mg} / \text { day }) \text { or } \\
\text { rosiglitazone } 4 \mathrm{mg} / \text { day or placebo with } \\
\text { glimepiride }(2-4 \mathrm{mg} / \text { day }) \text { on glycemic } \\
\text { control, body weight and safety in } \mathrm{T} 2 \mathrm{DM}\end{array}$ & $\begin{array}{l}\text { Double-blind } \\
\text { RCT }\end{array}$ & $\begin{array}{l}1,041 \text { patients, mean age } 56.2 \text { years, } \\
50 \% \text { male; study duration } 26 \text { weeks }\end{array}$ & $\begin{array}{l}\text { Liraglutide } 0.6,1.2 \text { or } 1.8 \mathrm{mg} \\
\text { and rosiglitazone placebo; } \\
\text { or liraglutide placebo and } \\
\text { rosiglitazone placebo; } \\
\text { or liraglutide placebo and } \\
\text { rosiglitazone } 4 \mathrm{mg} / \text { day }\end{array}$ \\
\hline $\begin{array}{l}\text { Nauck } \\
\text { et al. }[24] \\
2009\end{array}$ & $\begin{array}{l}\text { To compare the efficacy and safety of } \\
\text { liraglutide added to metformin with } \\
\text { addition of placebo or glimepiride to } \\
\text { metformin in patients previously treated } \\
\text { with oral antidiabetes therapy }\end{array}$ & $\begin{array}{l}\text { Double-blind } \\
\text { RCT }\end{array}$ & $\begin{array}{l}1,091 \text { patients, mean age } 56.6 \text { years, } \\
59 \% \text { male, mean duration of diabetes } \\
7.4 \text { years; study duration } 26 \text { weeks }\end{array}$ & $\begin{array}{l}\text { Liraglutide } 0.6,1.2 \text { or } \\
1.8 \text { mg q.d.; } \\
\text { glimepiride } 4 \text { mg q.d. }\end{array}$ \\
\hline $\begin{array}{l}\text { Seino } \\
\text { et al. [25], } \\
2008\end{array}$ & $\begin{array}{l}\text { To evaluate the dose-response efficacy and } \\
\text { safety of liraglutide in Japanese subjects } \\
\text { with T2DM }\end{array}$ & $\begin{array}{l}\text { Double-blind } \\
\text { RCT }\end{array}$ & $\begin{array}{l}226 \text { patients, mean age } 57.3 \text { years, } \\
57 \% \text { male, mean duration of diabetes } \\
7.6 \text { years; study duration } 14 \text { weeks }\end{array}$ & $\begin{array}{l}\text { Liraglutide } 0.1,0.3,0.6 \text { or } \\
0.9 \text { mg q.d. }\end{array}$ \\
\hline $\begin{array}{l}\text { Vilsboll } \\
\text { et al. [26], } \\
2007\end{array}$ & $\begin{array}{l}\text { To evaluate the efficacy and safety of } \\
\text { liraglutide in patients with T2DM }\end{array}$ & $\begin{array}{l}\text { Double-blind } \\
\text { RCT }\end{array}$ & $\begin{array}{l}165 \text { patients, mean age } 55.8 \text { years, } \\
61 \% \text { male, mean duration of diabetes } \\
5.5 \text { years; study duration } 14 \text { weeks }\end{array}$ & $\begin{array}{l}\text { Liraglutide } 0.65,1.25 \text { or } \\
1.90 \mathrm{mg}\end{array}$ \\
\hline $\begin{array}{l}\text { Russell-Jones } \\
\text { et al. [27], } 2008 \\
\text { (abstract only) }\end{array}$ & $\begin{array}{l}\text { To compare the efficacy and safety of } \\
\text { liraglutide, liraglutide placebo and open- } \\
\text { label insulin glargine }\end{array}$ & $\begin{array}{l}\text { Double-blind, } \\
\text { 26-week trial }\end{array}$ & 581 subjects & Liraglutide $1.8 \mathrm{mg}$ \\
\hline
\end{tabular}

RCT $=$ Randomized controlled trial.

\section{References}

-1 Rodbard HW, Blonde L, Braithwaite SS, Brett EM, Cobin RH, Handelsman Y, Hellman R, Jellinger PS, Jovanovic LG, Levy P, Mechanick JI, Zangeneh F: American Association of Clinical Endocrinologists medical guidelines for clinical practice for the management of diabetes mellitus. Endocr Pract 2007;13(suppl 1):1-68.

2 International Diabetes Federation: Diabetes Atlas. Brussels, IDF, 2010.

3 Centers for Disease Control and Prevention: National diabetes fact sheet: general information and national estimates on diabetes in the United States, 2007. Atlanta, US Department of Health and Human Services, Centers for Disease Control and Prevention, 2008.

4 American Diabetes Association: Economic costs of diabetes in the US in 2007. Diabetes Care 2008;31:596-615.

5 Diabetes in the UK 2009. Glasgow, 2009.
66 Philippe J, Raccah D: Treating type 2 diabetes: how safe are current therapeutic agents? Int J Clin Pract 2009;63:321-332.

7 European Medicines Agency: Tesavel (sitagliptin). European public assessment report (EPAR) summary for the public. EMEA/ 768259/2009. 2010.

8 European Medicines Agency: Merck Sharp \& Dohme limited summary of product characteristics: Januvia $100 \mathrm{mg}$ film-coated tablets. 2010.

9 Regional Drug and Therapeutics Centre: Sitagliptin. New Drug Evaluation No 85. 2007.

10 European Medicines Agency: Novartis summary of product characteristics: Galvus ${ }^{\mathrm{TM}}$ 2010.

11 European Medicines Agency: Eli Lilly \& Co summary of product characteristics: Byet$\mathrm{ta}^{\mathrm{TM}} .2010$.

12 European Medicines Agency: Novo Nordisk summary of product characteristics: Victo$\mathrm{za}^{\mathrm{TM}} \cdot 2010$.
13 Zinman B, Hoogwerf BJ, Duran GS, Milton DR, Giaconia JM, Kim DD, Trautmann ME, Brodows RG: The effect of adding exenatide to a thiazolidinedione in suboptimally controlled type 2 diabetes: a randomized trial. Ann Intern Med 2007;146:477-485.

14 De Fronzo RA, Ratner RE, Han J, Kim DD, Fineman MS, Baron AD: Effects of exenatide (exendin-4) on glycemic control and weight over 30 weeks in metformin-treated patients with type 2 diabetes. Diabetes Care 2005;28: 1092-1100.

15 Gao Y, Yoon KH, Chuang LM, Mohan V, Ning G, Shah S, Jang HC, Wu TJ, Johns D, Northrup J, Brodows R: Efficacy and safety of exenatide in patients of Asian descent with type 2 diabetes inadequately controlled with metformin or metformin and a sulphonylurea. Diabetes Res Clin Pract 2009;83: 69-76. 
- 16 Kendall DM, Riddle MC, Rosenstock J, 25 Seino Y, Rasmussen MF, Zdravkovic M, Zhuang D, Kim DD, Fineman MS, Baron $\mathrm{AD}$ : Effects of exenatide (exendin-4) on glycemic control over 30 weeks in patients with type 2 diabetes treated with metformin and a sulfonylurea. Diabetes Care 2005;28:10831091.

-17 Moretto TJ, Milton DR, Ridge TD, Macconell LA, Okerson T, Wolka AM, Brodows RG: Efficacy and tolerability of exenatide monotherapy over 24 weeks in antidiabetic drug-naive patients with type 2 diabetes: a randomized, double-blind, placebo-controlled, parallel-group study. Clin Ther 2008;30:1448-1460.

- 18 Poon T, Nelson P, Shen L, Mihm M, Taylor K, Fineman M, Kim D: Exenatide improves glycemic control and reduces body weight in subjects with type 2 diabetes: a dose-ranging study. Diabetes Technol Ther 2005;7:467477.

-19 Buse JB, Henry RR, Han J, Kim DD, Fineman MS, Baron AD: Effects of exenatide (exendin-4) on glycemic control over 30 weeks in sulfonylurea-treated patients with type 2 diabetes. Diabetes Care 2004;27:2628-2635.

-20 Fineman MS, Bicsak TA, Shen LZ, Taylor K, Gaines E, Varns A, Kim D, Baron AD: Effect on glycemic control of exenatide (synthetic exendin-4) additive to existing metformin and/or sulfonylurea treatment in patients with type 2 diabetes. Diabetes Care 2003;26: 2370-2377.

-21 Zinman B, Gerich J, Buse JB, Lewin A, Schwartz S, Raskin P, Hale PM, Zdravkovic M, Blonde L: Efficacy and safety of the human glucagon-like peptide-1 analog liraglutide in combination with metformin and thiazolidinedione in patients with type 2 diabetes (LEAD-4 Met + TZD). Diabetes Care 2009;32:1224-1230.

-22 Madsbad S, Schmitz O, Ranstam J, Jakobsen G, Matthews DR: Improved glycemic control with no weight increase in patients with type 2 diabetes after once-daily treatment with the long-acting glucagon-like peptide 1 analog liraglutide (NN2211): a 12-week, doubleblind, randomized, controlled trial. Diabetes Care 2004;27:1335-1342.

-23 Marre M, Shaw J, Brandle M, Bebakar WM, Kamaruddin NA, Strand J, Zdravkovic M, Le Thi TD, Colagiuri S: Liraglutide, a once-daily human GLP-1 analogue, added to a sulphonylurea over 26 weeks produces greater improvements in glycaemic and weight control compared with adding rosiglitazone or placebo in subjects with type 2 diabetes (LEAD-1 SU). Diabet Med 2009;26:268-278.

-24 Nauck M, Frid A, Hermansen K, Shah NS, Tankova T, Mitha IH, Zdravkovic M, During M, Matthews DR: Efficacy and safety comparison of liraglutide, glimepiride, and placebo, all in combination with metformin, in type 2 diabetes: the LEAD (liraglutide effect and action in diabetes)-2 study. Diabetes Care 2009;32:84-90.
Kaku K: Dose-dependent improvement in glycemia with once-daily liraglutide without hypoglycemia or weight gain: a doubleblind, randomized, controlled trial in Japanese patients with type 2 diabetes. Diabetes Res Clin Pract 2008;81:161-168.

26 Vilsboll T, Zdravkovic M, Le-Thi T, Krarup T, Schmitz O, Courreges JP, Verhoeven R, Buganova I, Madsbad S: Liraglutide, a longacting human glucagon-like peptide-1 analog, given as monotherapy significantly improves glycemic control and lowers body weight without risk of hypoglycemia in patients with type 2 diabetes. Diabetes Care 2007;30:1608-1610.

27 Russell-Jones D, Vaag A, Schmitz O, Sethi B, Lalic NM, Antic S: Significantly better glycaemic control/weight reduction with human GLP-1 analogue liraglutide, than with insulin glargine: all as add-on to metformin + sulphonylurea in type 2 diabetes. Diabetologia 2008;51:S68.

28 Scott R, Loeys T, Davies MJ, Engel SS: Efficacy and safety of sitagliptin when added to ongoing metformin therapy in patients with type 2 diabetes. Diabetes Obes Metab 2008; 10:959-969.

29 Aschner P, Kipnes MS, Lunceford JK, Sanchez M, Mickel C, Williams-Herman DE: Effect of the dipeptidyl peptidase-4 inhibitor sitagliptin as monotherapy on glycemic control in patients with type 2 diabetes. Diabetes Care 2006;29:2632-2637.

30 Charbonnel B, Karasik A, Liu J, Wu M, Meininger G: Efficacy and safety of the dipeptidyl peptidase-4 inhibitor sitagliptin added to ongoing metformin therapy in patients with type 2 diabetes inadequately controlled with metformin alone. Diabetes Care 2006;29:2638-2643.

31 Goldstein BJ, Feinglos MN, Lunceford JK, Johnson J, Williams-Herman DE: Effect of initial combination therapy with sitagliptin a dipeptidyl peptidase- 4 inhibitor, and metformin on glycemic control in patients with type 2 diabetes. Diabetes Care 2007;30: 1979-1987.

>32 Hanefeld M, Herman GA, Wu M, Mickel C, Sanchez M, Stein PP: Once-daily sitagliptin a dipeptidyl peptidase- 4 inhibitor, for the treatment of patients with type 2 diabetes. Curr Med Res Opin 2007;23:1329-1339.

33 Hermansen K, Kipnes M, Luo E, Fanurik D, Khatami H, Stein P: Efficacy and safety of the dipeptidyl peptidase- 4 inhibitor, sitagliptin, in patients with type 2 diabetes mellitus inadequately controlled on glimepiride alone or on glimepiride and metformin. Diabetes Obes Metab 2007;9:733-745.

34 Mohan V, Yang W, Son HY, Xu L, Noble L, Langdon RB, Amatruda JM, Stein PP, Kaufman KD: Efficacy and safety of sitagliptin in the treatment of patients with type 2 diabetes in China, India, and Korea. Diabetes Res Clin Pract 2009;83:106-116.
35 Nonaka K, Kakikawa T, Sato A, Okuyama K, Fujimoto G, Kato N, Suzuki H, Hirayama Y, Ahmed T, Davies MJ, Stein PP: Efficacy and safety of sitagliptin monotherapy in Japanese patients with type 2 diabetes. Diabetes Res Clin Pract 2008;79:291-298.

-36 Raz I, Chen Y, Wu M, Hussain S, Kaufman KD, Amatruda JM, Langdon RB, Stein PP, Alba M: Efficacy and safety of sitagliptin added to ongoing metformin therapy in patients with type 2 diabetes. Curr Med Res Opin 2008;24:537-550

-37 Scott R, Wu M, Sanchez M, Stein P: Efficacy and tolerability of the dipeptidyl peptidase-4 inhibitor sitagliptin as monotherapy over 12 weeks in patients with type 2 diabetes. Int J Clin Pract 2007;61:171-180.

>38 Rosenstock J, Brazg R, Andryuk PJ, Lu K, Stein P: Efficacy and safety of the dipeptidyl peptidase-4 inhibitor sitagliptin added to ongoing pioglitazone therapy in patients with type 2 diabetes: a 24 -week, multicenter, randomized, double-blind, placebo-controlled, parallel-group study. Clin Ther 2006;28:1556-1568.

>39 Raz I, Hanefeld M, Xu L, Caria C, WilliamsHerman D, Khatami H: Efficacy and safety of the dipeptidyl peptidase- 4 inhibitor sitagliptin as monotherapy in patients with type 2 diabetes mellitus. Diabetologia 2006;49: 2564-2571.

40 Ahren B, Gomis R, Standl E, Mills D, Schweizer A: Twelve- and 52-week efficacy of the dipeptidyl peptidase IV inhibitor LAF237 in metformin-treated patients with type 2 diabetes. Diabetes Care 2004;27:2874-2880.

41 Ristic S, Byiers S, Foley J, Holmes D: Improved glycaemic control with dipeptidyl peptidase- 4 inhibition in patients with type 2 diabetes: vildagliptin (LAF237) dose response. Diabetes Obes Metab 2005;7:692698.

42 Bosi E, Camisasca RP, Collober C, Rochotte E, Garber AJ: Effects of vildagliptin on glucose control over 24 weeks in patients with type 2 diabetes inadequately controlled with metformin. Diabetes Care 2007;30:890-895.

$\checkmark 43$ Dejager S, Razac S, Foley JE, Schweizer A: Vildagliptin in drug-naive patients with type 2 diabetes: a 24 -week, double-blind, randomized, placebo-controlled, multipledose study. Horm Metab Res 2007;39:218223.

44 Garber AJ, Schweizer A, Baron MA, Rochotte E, Dejager S: Vildagliptin in combination with pioglitazone improves glycaemic control in patients with type 2 diabetes failing thiazolidinedione monotherapy: a randomized, placebo-controlled study. Diabetes Obes Metab 2007;9:166-174.

45 Garber AJ, Foley JE, Banerji MA, Ebeling P, Gudbjornsdottir S, Camisasca RP, Couturier A, Baron MA: Effects of vildagliptin on glucose control in patients with type 2 diabetes inadequately controlled with a sulphonylurea. Diabetes Obes Metab 2008;10:10471056. 
46 Goodman M, Thurston H, Penman J: Efficacy and tolerability of vildagliptin in patients with type 2 diabetes inadequately controlled with metformin monotherapy. Horm Metab Res 2009;41:368-373.

-47 Kikuchi M, Abe N, Kato M, Terao S, Mimori N, Tachibana H: Vildagliptin dose-dependently improves glycemic control in Japanese patients with type 2 diabetes mellitus. Diabetes Res Clin Pract 2009;83:233-240.

-48 Pi-Sunyer FX, Schweizer A, Mills D, Dejager S: Efficacy and tolerability of vildagliptin monotherapy in drug-naive patients with type 2 diabetes. Diabetes Res Clin Pract 2007;76:132-138.
9 Scherbaum WA, Schweizer A, Mari A, Nilsson PM, Lalanne G, Wang Y, Dunning BE, Foley JE: Evidence that vildagliptin attenuates deterioration of glycaemic control during 2-year treatment of patients with type 2 diabetes and mild hyperglycaemia. Diabetes Obes Metab 2008;10:1114-1124.

50 Mimori N, Terao S, Holmes D. Vildagliptin improves glucose control as evidenced by $\mathrm{A}_{1 \mathrm{c}}$ therapy after 12 weeks therapy in Japanese patients with type 2 diabetes. Diabetes 2006; 55(suppl 1):A125.

51 Egger M, Davey Smith G: Meta-analysis: potentials and promise. BMJ 1997;315:13711374 .
52 Chia CW, Egan JM: Incretin-based therapies in type 2 diabetes mellitus. J Clin Endocrinol Metab 2008;93:3703-3716.

53 Canadian Diabetes Association Clinical Practice Guidelines Expert Committee: Canadian Diabetes Association 2003 clinical practice guidelines for the prevention and management of diabetes in Canada. Can J Diabetes 2003;27:1-152.

54 Russell-Jones D, Khan R: Insulin-associated weight gain in diabetes - causes, effects and coping strategies. Diabetes Obes Metab 2007;9:799-812. 